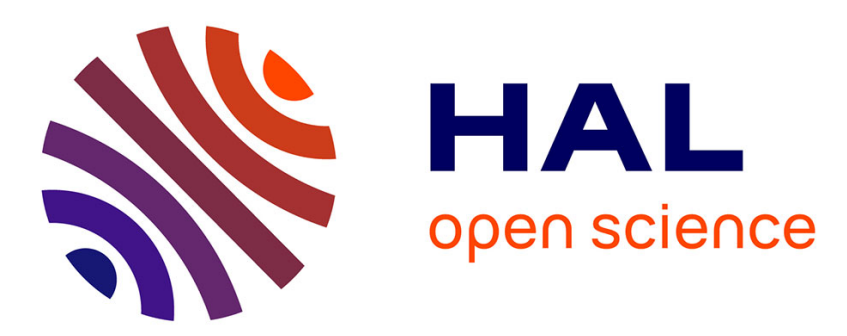

\title{
Unusual, pH-Induced, Self-Assembly Of Sophorolipid Biosurfactants
}

Niki Baccile, Florence Babonneau, Jacques Jestin, Gerard Pehau-Arnaudet, Inge van Bogaert

\section{- To cite this version:}

Niki Baccile, Florence Babonneau, Jacques Jestin, Gerard Pehau-Arnaudet, Inge van Bogaert. Unusual, pH-Induced, Self-Assembly Of Sophorolipid Biosurfactants. ACS Nano, 2012, 6 (6), pp.47634776. 10.1021/nn204911k . hal-01455100

\section{HAL Id: hal-01455100 \\ https://hal.sorbonne-universite.fr/hal-01455100}

Submitted on 3 Feb 2017

HAL is a multi-disciplinary open access archive for the deposit and dissemination of scientific research documents, whether they are published or not. The documents may come from teaching and research institutions in France or abroad, or from public or private research centers.
L'archive ouverte pluridisciplinaire HAL, est destinée au dépôt et à la diffusion de documents scientifiques de niveau recherche, publiés ou non, émanant des établissements d'enseignement et de recherche français ou étrangers, des laboratoires publics ou privés. 


\title{
Unusual, pH-Induced, Self-Assembly Of Sophorolipid Biosurfactants
}

\section{Authors}

Niki Baccile, ${ }^{\mathrm{a}, \mathrm{b}, \mathrm{c} *}$ Florence Babonneau, ${ }^{\mathrm{a}, \mathrm{b}, \mathrm{c}}$ Jacques Jestin, ${ }^{\mathrm{d}}$ Gerard Pehau-Arnaudet, ${ }^{\mathrm{e}}$ Inge Van Bogaert $^{\mathrm{f}}$

\section{Affiliations}

a-UPMC Univ Paris 06, UMR 7574, Chimie de la Matière Condensée de Paris, F-75005, Paris, France. E-mail : niki.baccile@upmc.fr

b- CNRS, UMR 7574, Chimie de la Matière Condensée de Paris, F-75005, Paris, France c - Collège de France, UMR 7574, Chimie de la Matière Condensée de Paris, F-75005, Paris, France

d. Laboratoire Léon Brillouin, LLB, CEA Saclay, F-91191 Gif-sur-Yvette Cedex, France

e. Plate-Forme de Cryomicroscopie Moléculaire, URA 2185, Institut Pasteur, 28 Rue du Docteur Roux, F-75015, Paris, France

f. InBio, Department of Biochemical and Microbial Technology, Faculty of Bioscience Engineering, Ghent University, Coupure Links 653, 9000, Ghent, Belgium

\section{Keywords}

Self-assembly, biosurfactants, microbial-derived surfactants, sophorolipids, small angle neutron scattering, cryo-TEM

\begin{abstract}
An increasing need exists nowadays for simple, bio-derived, non-toxic and up-scalable compounds with stimuli-responsive properties. Acidic sophorolipids (SL) are glucose-based biosurfactants derived from the yeast broth of Candida bombicola (teleomorph: Starmerella bombicola). The specific design of this molecule, a sophorose head with a free end-COOH group at the end of the alkyl chain, makes it a potentially interesting $\mathrm{pH}$-responsive
\end{abstract}


compound. We have specifically investigated this assumption using a combination of small angle neutron scattering (SANS), transmission electron microscopy under cryogenic conditions (Cryo-TEM) and nuclear magnetic resonance (NMR) techniques and found a strong dependence of SL self-assembly on the degree of ionization, $\alpha$, of the COOH group at concentration values as low as $5 \mathrm{wt} \%$ and $0.5 \mathrm{wt} \%$. At least three regimes can be identified where the supramolecular behaviour of SL is unexpectedly different: 1) at low $\alpha$ values, selfassembly is driven by concentration, $\mathrm{C}$, and micelles are mainly identified as non-ionic objects whose curvature decreases (sphere-to-rod) with C; 2) at mid $\alpha$-values, the formation of $\mathrm{COO}^{-}$groups introduces negative charges at the micellar surface inducing an increase in curvature (rod-to-sphere transition). Repulsive electrostatic long-range interactions appear at this stage. In both regime 1 and 2 the cross-section radius of the micelles is below $25 \AA$. This behaviour is concentration independent. 3) At $\alpha=1$, individual micelles seem to disrupt to favour the formation of large net-like tubular aggregates whose size is above $100 \mathrm{~nm}$. Such a complex behaviour is very unique as it is generally not observed for common alkyl-based surfactants in the concentration range below 5-10 wt\%.

Keywords: sophorolipids, microbial glycolipids, self-assembly, biosurfactants, small-angle neutron scattering (SANS), ionization degree, $\mathrm{pH}$-responsive

Stimuli-responsive surfactants are a class of compounds which have recently attracted major interest in polymer chemistry and material science. The control of the aggregation state, phase behaviour and surface tension at will has stimulated much effort both in fundamental and applied science. Stabilization of emulsions, suspensions or foams, drug encapsulation and delivery, hard-surface cleaning, personal care applications, surface charge variations, wettability control, enhancement of viscoelastic properties, recyclability, heat-transfer fluids, drag-reduction agents and dynamic templates for nanomaterial synthesis are just some of the possible applications while temperature, electric field, ionic strength and $\mathrm{pH}$ are commonly studied "switches". pH, in particular, is a simple tool that induces different aggregation states of a given molecule. Classical commercial surfactants are either ionic (cationic, anionic, zwitterionic) or non-ionic and do not show a strong $\mathrm{pH}$-sensitive behaviour. Actually, for some ester and amide-based compounds, $\mathrm{pH}$ represents a source of molecular disruptor. Block copolymers have larger sizes, good thermal stability, and are promising tools in drug delivery applications due to their high loading capacity of active compounds; additionally, some of 
them have very interesting $\mathrm{pH}$-responsive properties. In particular, poly(acrylic acid), PAA, based block copolymers are known to form pH-sensitive nanostuctures. ${ }^{1,2}$ Nevertheless, their physico-chemical behaviour is strongly dependent on the chemical nature and size of the hydrophobic block; for example, adsorption and assembly kinetics are quite slow for poly(styrene), PS, or poly(n-butyl acrylate), PBA, based blocks and that is the case for many block copolymer-based systems; for this reason, the interest to find $\mathrm{pH}$-responsive systems based on small amphiphiles is still high. Common surfactants made of an aliphatic chain (e.g., CTAB, SDS, Brij ${ }^{\circledR}$, etc...) show typical lyotropic properties but no specific pH-driven assembly effects, exception made for few compounds, like oleyldimethyamine oxide ${ }^{3}$ and some interesting cases of binary systems. In two recent examples, cetyltrimethylammonium bromide, CTAB, used in presence of potassium phtalic acid ${ }^{4}$ or $N$-erucamidopropyl- $N, N$,dimethylamine combined with maleic acid ${ }^{5}$ have displayed the formation ofpH-induced elongated wormlike micelles, which are interesting systems for their viscoelastic properties. Drug-delivery applications were also targeted in binary monolinolein/phloroglucinol systems ${ }^{6}$ but less importance was given to a specific study on the self-assembly mechanism. Furthemore, bolaform surfactants are also a wide family of compounds that contain double hydrophilic units symmetrically located at each end of a hydrophobic spacer. Interesting $\mathrm{pH}$ responsive aggregation properties have been observed on specific, well-designed, molecules, such as asymmetric bolaamphiphiles, but their laborious synthesis procedure may prevent their large-scale employment. ${ }^{7-9}$

In this context, a new, emerging, field on bio-based surfactants has been intensively studies in the past 20 years to fulfil the expectations of replacing non-renewable, mineral oilderived, products ${ }^{10}$ in agreement with the 12 principles of green chemistry. Glycolipids are non-ionic surface active agents composed of a hydrophilic carbohydrate-based head linked to a fatty acid or a fatty alcohol through an ester, an amide or an ether bond. ${ }^{11}$ They already replace, and/or are destined to massively replace, classical surfactant families like SDS or $\mathrm{CTAB}$ in home and personal care products. For these reasons, their physico-chemical properties have been studied in the past 15 years. Effect of molecular structure (e.g., chain length, number of sugar units), concentration, use of co-solvents (short-chain alcohol, for instance), co-surfactants on the critical micelle concentration ( $\mathrm{cmc}$ ), surface tension, rheology, cleaning power but also self-assembly and mesophase formation have shown particular perspectives for many of these compounds. ${ }^{11-13}$ These molecules are generally synthesized via several chemical strategies, reviewed by von Rybinski, ${ }^{11}$ from renewable compounds like fatty acids and sugars. In terms of self-assembly, sugar-based surfactants show classical 
lyotropic behaviour, as expected for ionic or non-ionic compounds but no specific stimulusresponsive property has been attributed to them till date. In addition, they are still much more expensive in their pure form with respect to classical surfactants; in fact, low-cost commercial alkylpolyglucosides (APG) are actually sold as formulations presenting either a non-constant number of glucose units or mixtures of at least two different chain lengths. For these reasons, their use in nanoscience-related research topics has not been highly explored, yet.

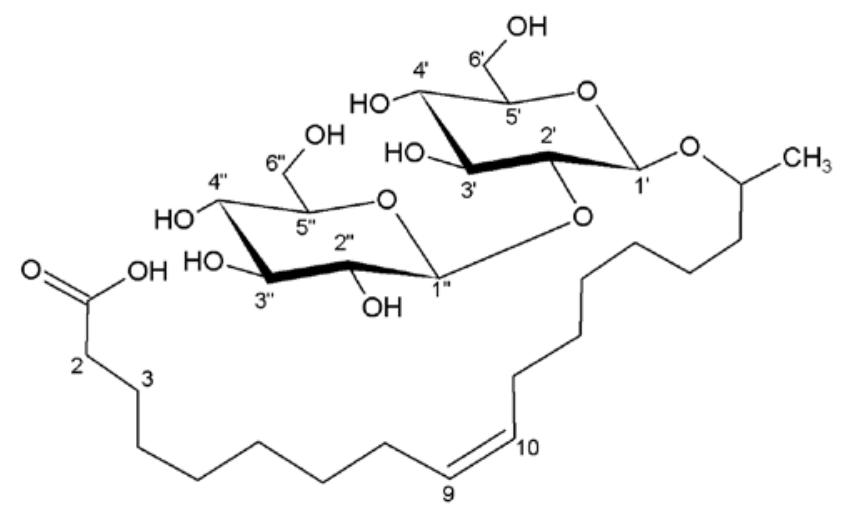

Figure 1 - Acidic form of sophorolipids (SL) obtained from Candida bombicola

In a recent communication, ${ }^{14}$ we have investigated the self-assembly and structuring properties of acidic sophorolipids (Figure 1), a family of entirely bio-derived glycolipids. These natural compounds can be obtained in large amounts by yeast culture in presence of several carbon sources, like glucose, fatty acids but even alkanes and waxes. ${ }^{15-18}$ Due to their reduced environmental impact biosynthesis, confirmed by a recent life cycle analysis study, ${ }^{19}$ sophorolipids (SL) have attracted a fair attention of the home and skin-care industry. ${ }^{20-22}$ Nevertheless, few recent studies have highlighted interesting anticancer ${ }^{23}$ or self-assembly properties. ${ }^{14,18}$ Nonetheless, the limited number of works involving SL has prevented a deeper knowledge and understanding of their physico-chemical properties. Acidic SL are composed of a sophorose unit attached to an oleic acid moiety through an ether bond on the C17 carbon atom of the fatty acid chain. This particular feature leaves the $\mathrm{COOH}$ group unaffected in contrast with most fatty acid-derived surfactant obtained by chemical synthesis and in which the carboxylic group is generally engaged in an ester bond. Acidic SL are water-soluble and the $\mathrm{COOH}$ group constitutes a good $\mathrm{pH}$-responsive probe.

Zhou et al. ${ }^{18}$ were the first ones to show the supramolecular assembling properties of $\mathrm{SL}$ in water supplying evidence of both $\mathrm{pH}$ and time factors. In our recent work, ${ }^{14}$ we focused on the self-assembly of SL into different micellar objects (spherical, cylindrical and wormlike) and we have shown how they can be exploited as porogenic agents in the synthesis of silica thin films. In this study, we provide more details on the behaviour of SL in aqueous 
medium by demonstrating how their shape, long-range interactions, surface charge and aggregate dimensions are governed by $\mathrm{pH}$. In the domain of bio-engineered glycolipids, only rhamnolipids were reported to have a pH-dependent behaviour, their morphology changing from vesicles to micelles in the region $4.5<\mathrm{pH}<7.0 .^{24}$

In a recent review on the self-assembly behaviour of biosurfactants, Kitamoto et al. ${ }^{25}$ focused on many compounds but sophorolipids were excluded. The study of simple, sustainable, bio-derived, monocomponent, stimuli-responsive and up-scalable molecular systems give new perspectives in the scientific context described above. In addition, their close molecular similarity to specific nanotube-forming asymmetric bolaform surfactants obtained through chemical engineering suggests that their self-assembly behaviour is up to now largely under-evaluated. ${ }^{26}$

In this work, we propose a detailed study on the $\mathrm{pH}$-responsive properties of the open acidic form of SL in water in the $\mathrm{pH}$ range between 4.5 and 11, corresponding to a degree of ionization, ${ }^{27} \alpha$, between 0.2 and 1 at concentration values below $5 \mathrm{wt} \%$. We show here, using a combination of small angle neutron scattering (SANS), cryo-TEM and NMR experiments that variations in the degree of ionization of SL will introduce negative charges at the micellar surface inducing changes in their shape, aggregation state and surface properties. In particular, SANS is a very powerful technique to study soft matter behaviour in solution. Figure 2 shows a schematic representation of the main pieces of information that can be extracted from a loglog representation of a SANS spectrum. $\mathrm{I}(\mathrm{q})$ is the scattered neutron intensity in absolute scale as a function of the wavevector $\mathrm{q} ; \mathrm{P}(\mathrm{q})$ is the form factor and $\mathrm{S}(\mathrm{q})$ the structure factor. For isolated objects $\mathrm{S}(\mathrm{q})$ being unitary and the analysis of slope of $\mathrm{I}(\mathrm{q})$ (in log-log scale) provides information on the micellar shape; in presence of repulsive interactions between well-defined objects, the structure factor cannot be neglected anymore in the intermediate q-range and it strongly contributes, generally with a scattering peak, to the overall I(q) while it tends to a unitary value at high q. In case of undefined shapes and sizes, as for fractal objects, one observes an undefined increase of the scattered intensity at low q values, whose slope, in some cases, can be related to the fractal dimension of the object. 


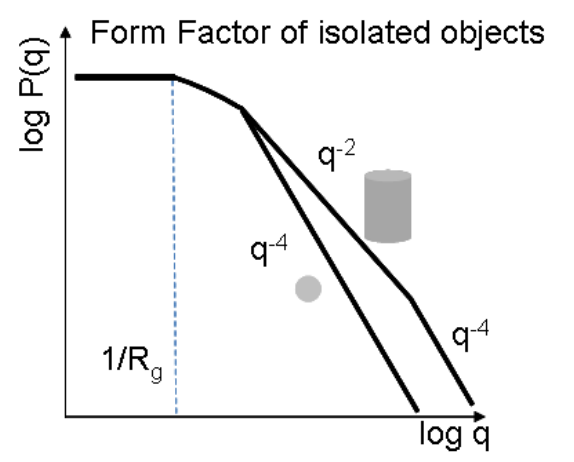

$I(q)$ : Intensity in absolute scale $\left(\mathrm{cm}^{-1}\right)$

$P(q)$ : Form Factor

$S(q)$ : Structure Factor

$\mathrm{I}(\mathrm{q}) \sim \mathrm{P}(\mathrm{q}) \times \mathrm{S}(\mathrm{q})$
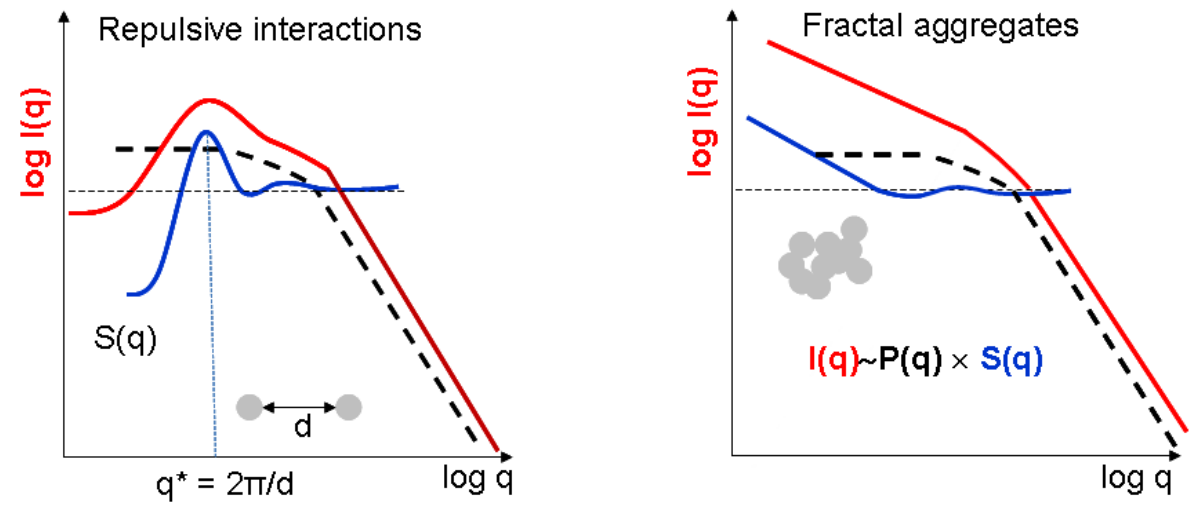

Figure 2 - Schematic log-log representation of the typical neutron scattered intensity (I(q)) under diluted (isolated objects), interactive and fractal regimes.

In this particular study, SANS spectra clearly show the existence of at least three different regions where supramolecular SL aggregates are unexpectedly different. In contrast to many similar systems, whose rich nature of supramolecular assemblies is mostly driven by concentration effects above 5-10 wt\%, we find here structures as different as nanometer-sized spherical micelles and large net-like aggregates of several hundred of nanometers can form when passing from $\alpha=0.2$ to $\alpha=1$.

\section{Results and discussion}

In a recent communication ${ }^{14}$ we have shown that at $\mathrm{pH}<5$ concentration of the acidic SL influences the shape of micellar geometry, going from spherical (5 mg/mL) to wormlike objects $(220 \mathrm{mg} / \mathrm{mL})$ in the self-assembly process. Additionally, we supply evidence of the fact that $\mathrm{pH}$ seems to have an effect on the long-range interactions between micelles at concentration values as low as $50 \mathrm{mg} / \mathrm{mL}$. In the next sections, we will discuss in more detail the self-assembly behaviour of SL as a function of $\mathrm{pH}$.

Concentration effects. In Figure 3, we show SANS experiments of SL in $\mathrm{D}_{2} \mathrm{O}$ at $4<\mathrm{pH}<5$ as a function of SL concentration, which was increased up to $500 \mathrm{mg} / \mathrm{mL}$. Data are 
normalized by the sample concentration, C, to which the cmc value of SL is subtracted in order to consider only the micellar contribution to the scattered signal. At low concentrations, $\mathrm{C}=5$ and $50 \mathrm{mg} / \mathrm{mL}$, the signal can be fitted using the form factor, $\mathrm{P}(\mathrm{q})$, of, respectively, a sphere and a cylinder, as schematized in Figure 2 and actually described in a previous communication. ${ }^{14}$ However, different form factors can also be employed in good approximation, as summarized in Table S1 in the Supporting information. At $5 \mathrm{mg} / \mathrm{mL}$, a spherical or ellipsoidal model can be applied with typical radii around $\mathrm{R}=26.1 \AA$ for the sphere and $\mathrm{R}=15.4 \AA$ for the ellipsoid and a typical length of $\mathrm{L}=61.4 \AA$ for the latter. Polydispersity seems to play an important role for these systems, values as high as $30 \%$ should be employed in order to fit the experimental data. At $50 \mathrm{mg} / \mathrm{mL}$, the cylindrical model is by far the best suited where $\mathrm{R}=14.2 \AA$ and $\mathrm{L}=139.0 \AA$ with a polydispersity of $18 \%$. A vesicle form factor can also be used but the polydispersity increases up to $50 \%$, which does not seem to be a reasonable value. The corresponding fits are given in Figure S1 in the Supporting information.

When increasing the concentration up to $500 \mathrm{mg} / \mathrm{mL}$, a decrease in the absolute intensity at low- $q$ values occurs, as expected for more condensed matter. At the same time, a very weak and broad interaction peak is detected at approximately $q=8 \cdot 10^{-2} \AA^{-1}$, corresponding to an intermicellar distance, d, of about $70 \AA$ : a non-unitary structure factor, S(q), contributes then to $\mathrm{I}(\mathrm{q})$, as also schematized in Figure 2. At this concentration, micelles are most likely constituted of entangled wormlike objects, ${ }^{14}$ but if one considers that the measured crosssection of the cylinders is approximately $30 \AA$, it can be deduce that they are not in direct contact, but rather with an average micelle-to-micelle distance of the order of $40 \AA$. Despite the relatively high SL concentration in solution, repulsive long-range intermicellar interactions do not seem to play a major role.

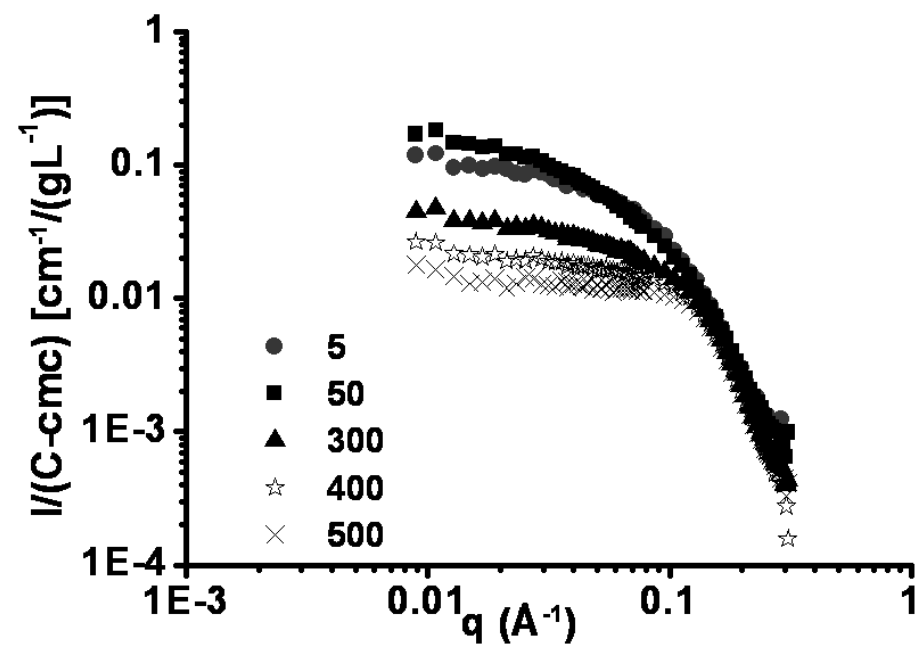


Figure 3 - Log-log representation of I(q) vs. q of SANS experiments performed on SL/D ${ }_{2} \mathrm{O}$ at different concentrations. Values are given in $\mathrm{mg} / \mathrm{mL}$. Intensity is concentration-scaled and normalized by the SL cmc. $4<$ pH $<5$.

pH effects. The role of $\mathrm{pH}$ on the self-assembly of SL in solution was briefly shown by Zhuo et al. ${ }^{18}$ Using optical microscopy and FT-IR arguments, they reported the formation of shortrange interacting micelles at $\mathrm{pH}=7.8$ and, for concentrations above $1 \mathrm{mg} / \mathrm{mL}$, the formation of large aggregates (radius of gyration, $\mathrm{Rg}>100 \mathrm{~nm}$ ). Nevertheless, these techniques are less adapted to characterize nanometer-scale systems with respect to small angle scattering techniques. In our previous work, SANS experiments demonstrates that long-range micellar interactions can develop as a function of $\mathrm{pH}$ at a given concentration, but no details were given about the nature of these interactions. Hereafter, we go deeper in the understanding of these phenomena. In Figure 4a,b we report the log-log evolution of SANS intensity recorded on SL solution at different $\mathrm{pH}$ values for two concentrations: $5 \mathrm{mg} / \mathrm{mL}$ (0.5 wt\%) (Figure 4a) and $50 \mathrm{mg} / \mathrm{mL}$ (5 wt\%) (Figure $4 \mathrm{~b}$ ). The differences among the low-angle scattering curves allow us to identify at least three different regimes.
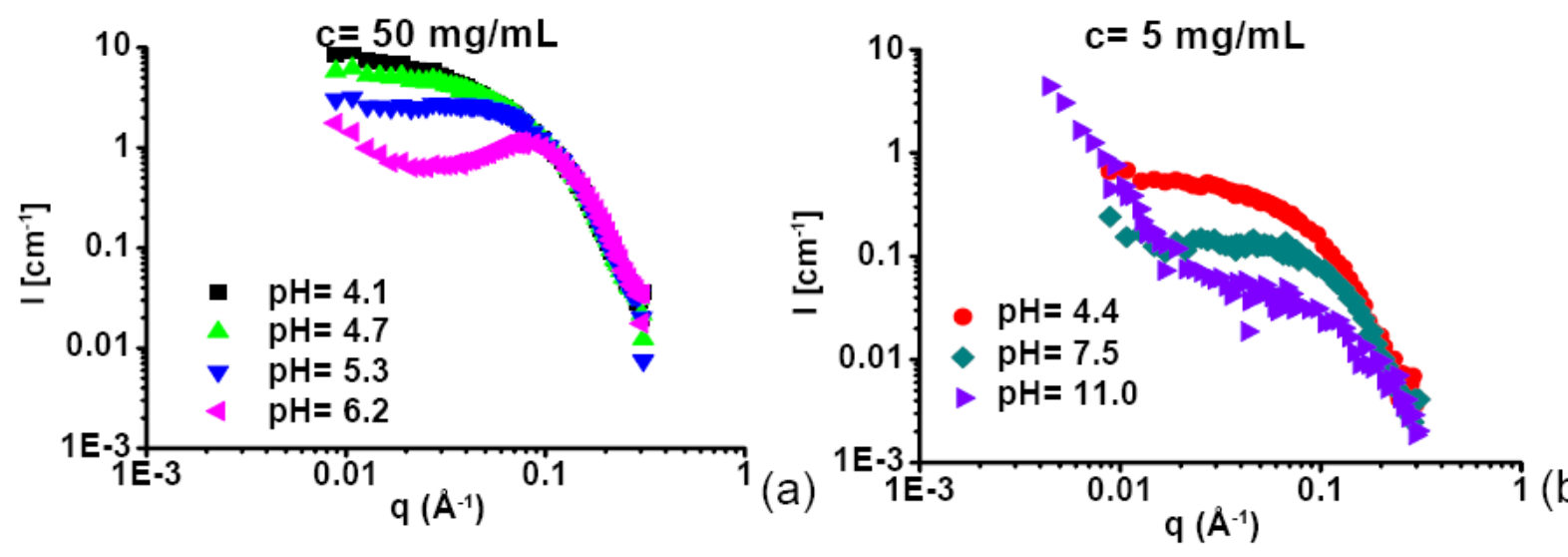

Figure 4 - log-log representation of I(q) for SL solutions at (a) $50 \mathrm{mg} / \mathrm{mL}$ and (b) $5 \mathrm{mg} / \mathrm{mL}$ at increasing pH values.

- Regime $1(\mathrm{pH}<5)$. Increase in concentration drives micellar shape from spherical (red circles) to cylinders (black squares) to wormlike (see ref 14).

- Regime $2(5<\mathrm{pH}<8)$. The effect of $\mathrm{pH}$ on self-assembly in this $\mathrm{pH}$ range is reflected on the long-range micellar interactions. A broad, but strong interaction peak grows in the 50 $\mathrm{mg} / \mathrm{mL}$ system due to a non-unitary structure factor, $\mathrm{S}(\mathrm{q})$ (Figure $4 \mathrm{a}$ ), as also schematized in Figure 2. The spectrum at $\mathrm{pH}=6.2$ is also followed by an increase in intensity at low- $q$ values. Both phenomena, at a less extent, are also observed on the $5 \mathrm{mg} / \mathrm{mL}$ solution at $\mathrm{pH}=7.5$ (Figure 4b). 
- Regime $3(\mathrm{pH}>8)$. Higher $\mathrm{pH}$ values seem to provoke a disruption of intermicellar interactions, as shown by the loss of the characteristic peak in the $5 \mathrm{mg} / \mathrm{mL}$ system (Figure 4b) at $\mathrm{pH}=11.0$. On the contrary, a very strong increase in $\mathrm{I}(\mathrm{q})$ occurs at $\mathrm{q}<0.03 \AA^{-1}$ indicating the existence of large aggregates, whose interesting nature will be described later.

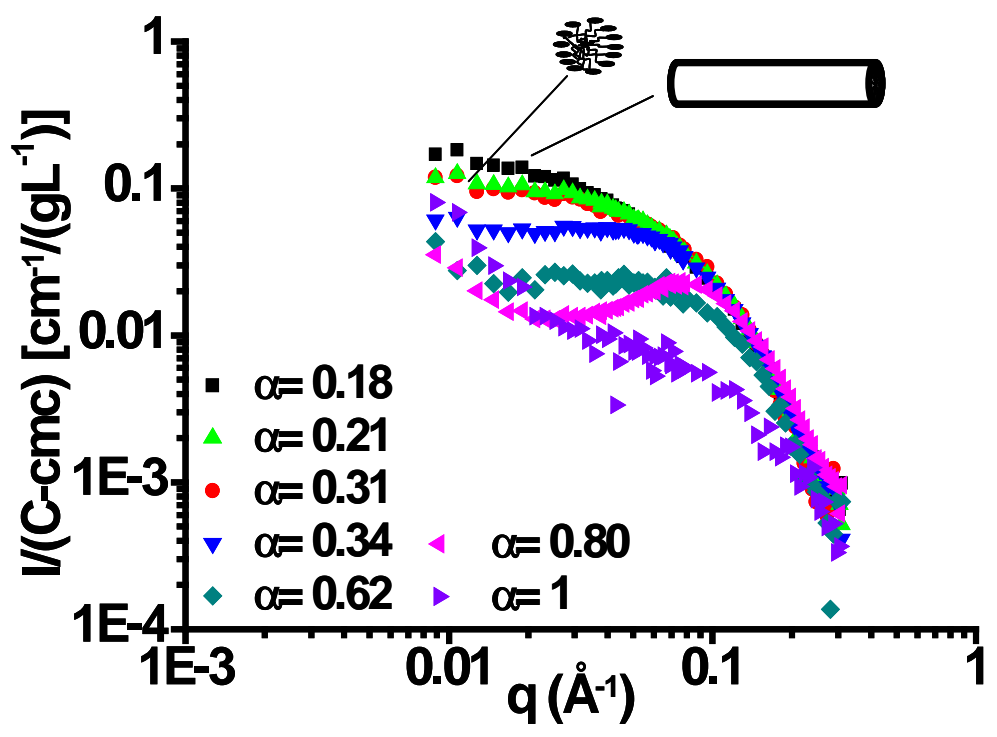

Figure 5 - Concentration and cmc scaled data presented in Figure 4 as a function of the ionization degree, $\alpha$, calculated according to Eq.1. The scheme summarizes the effect of concentration and charge on the shape of sophorolipid micelles. Details about the fits and derived micellar geometrical data can be found in Figure S1, Figure S2, Table S1 in Supporting information and Table 1.

If compared to non-ionic glycolipids, SL have unexpected features; their $\mathrm{pH}$-responsive behaviour derives from the free $\mathrm{COOH}$ group, and its degree of ionization, which plays a major role in the overall self-assembling properties. The amount of residual $\mathrm{COO}^{-}$in SL upon increasing $\mathrm{pH}$ can be estimated by adding the fraction of self-dissociated $\mathrm{COOH}$ at equilibrium, $\left[\mathrm{COO}^{-}\right]_{\text {sd }}$ (sd stands for self-dissociation). The former can be estimated by the dissociation constant of oleic acid (pKa of oleic acid is $4.8^{28,29}$ ) in water and after determining its equilibrium $\mathrm{pH}$, and the amount of added sodium hydroxyde, used to neutralize the carboxylic acid groups. The concentration of $\mathrm{COO}^{-},\left[\mathrm{COO}^{-}\right]_{\mathrm{NaOH}}$, is then directly proportional to the molar concentration of $\mathrm{NaOH}$. In this case, it is interesting to consider the overall degree of ionization, $\alpha$, defined in Eq. 1 :

$$
\alpha=\frac{\left[\mathrm{COO}^{-}\right]_{s d}+\left[\mathrm{COO}^{-}\right]_{\mathrm{NaOH}}}{[\mathrm{COOH}]_{\mathrm{SL}}}
$$


From now on, we will refer to all experiments in terms of the SL ionization degree and spectra presented in Figure 4a,b can be normalized with respect to the SL concentration (5 and $50 \mathrm{mg} / \mathrm{mL}$ ), as shown in Figure 5. Please note that, even if now data can be discussed as a function of $\alpha$, which is done afterwards, unexpected effects due to concentration may occur and a systematic study is eventually needed. Hereafter we rather focus on a new, phenomenological, self-assembling behaviour to which sophorolipids are submitted under the selected conditions.

Data presented in Figure 5 suggest that $\alpha$ strongly drives the self-assembly of SL from regime 1 through regime 3 with limited influence on concentration, at least for the tested values. At $\alpha>0.3$, a transition occurs between regime 1 and 2 while one has to reach at least $\alpha=1$ to be in regime 3.

Table 1 - Results from fits of SANS data shown in Figure $S 1(\alpha=0.31 ; \alpha=0.18)$ and Figure $S 2$ in the Supporting information

\begin{tabular}{|c|c|c|c|c|c|}
\hline $\mathrm{C}(\mathrm{mg} / \mathrm{mL})$ & $\alpha$ & Form Factor & $\mathbf{R}(\AA)$ & $\sigma$ & L ( $(\AA)$ \\
\hline \multirow{4}{*}{5} & \multirow{2}{*}{0.31} & Sphere & 26.1 & 0.30 & - \\
\hline & & Ellipsoid & 15.4 & 0.21 & 61.4 \\
\hline & & & & & \\
\hline & 1 & Sphere & 16.0 & 0.30 & - \\
\hline \multirow{3}{*}{50} & 0.18 & Cylinder & 14.2 & 0.18 & 139.0 \\
\hline & \multirow{2}{*}{0.21} & Sphere & 30.7 & 0.40 & - \\
\hline & & Ellipsoid & 15.0 & 0.29 & 77.4 \\
\hline
\end{tabular}

Table 1 shows the values of the fits of SANS data for SL solutions at $\mathrm{C}=5 \mathrm{mg} / \mathrm{mL}$ and 50 $\mathrm{mg} / \mathrm{mL}$ at different $\alpha$ values. Interestingly, for the $50 \mathrm{mg} / \mathrm{mL}$ system, when $\alpha$ increases from 0.18 to 0.21 , spherical $(\mathrm{R}=26.1 \AA)$ or ellipsoidal $(\mathrm{R}=15.0 \AA$; $\mathrm{L}=77.4 \AA)$ geometry models must be employed instead of the cylindrical one. The same models must be used to fit the lower concentration system, $C=5 \mathrm{mg} / \mathrm{mL}$, where micelles are spherical up to $\alpha=0.31$. At $\alpha=1$, fit of the full data set becomes challenging but an estimation of the micellar crosssection can be done by fitting only the high-q data region. A sphere form factor can be employed, as shown in Figure S2 in the Supporting information, providing $\mathrm{R}=16.0 \AA$ (Table 1). Nevertheless, a specific insight on the nature of the aggregates obtained at $\alpha=1$ conditions will be given later. 
The shape of SL micelles is highly influenced by the $\mathrm{pH}$ and data above show that, at a given concentration and increasing ionization degree, the micellar curvature increases because of a cylinder to ellipsoid/sphere transition, as shown by comparing, at $50 \mathrm{mg} / \mathrm{mL}$, the system at $\alpha=$ 0.18 and $\alpha=0.21$. In addition, as expected, the size of the SL micelle increases when passing from the low to high concentration system (Table 1). In the spherical model hypothesis, the radius goes from $26.1 \AA(5 \mathrm{mg} / \mathrm{mL})$ to $30.7 \AA$ (50 mg/mL) while in the ellipsoid model, the radius is practically constant $(\mathrm{R}=15.0 \AA)$ but the length varies from $61.4 \AA(5 \mathrm{mg} / \mathrm{mL})$ to 77.4 $\AA$ (50 mg/mL). When decreasing the value of $\alpha$ at $50 \mathrm{mg} / \mathrm{mL}$, the length of the cylinder goes from $77.4 \AA$ to $139.0 \AA$.

These data will be discussed deeply later on in the manuscript, one can say already state that SL have interesting $\mathrm{pH}$-responsive self-assembling properties in water. Nevertheless, many questions are still open and in particular on the nature of the interacting aggregates in regime 2 and regime 3.

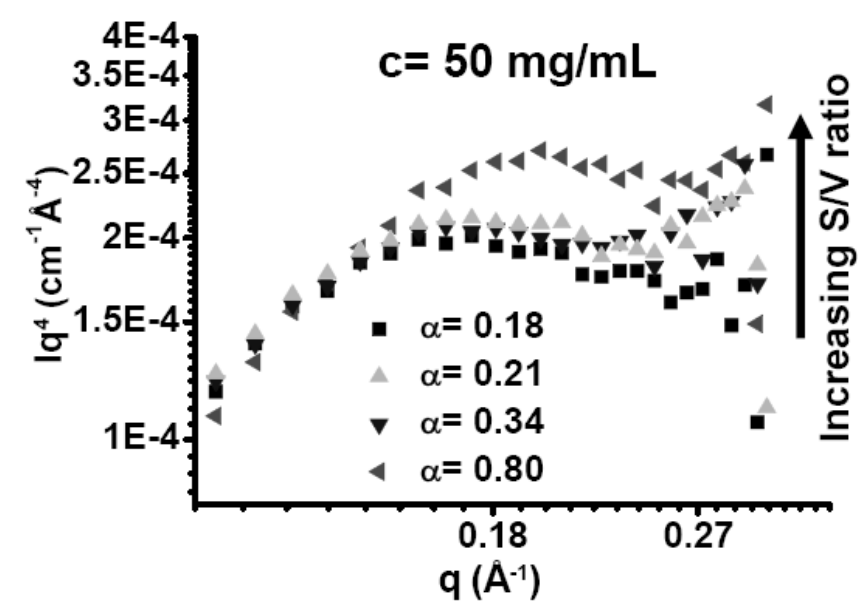

Figure 6 - Porod plot $\left[I \cdot q^{4}(q)\right]$ of SL solutions at $50 \mathrm{mg} / \mathrm{mL}$ at four different values of $\alpha$. S/V stands for surface-to-volume ratio

SL behaviour in Regime 2. At the concentration of $50 \mathrm{mg} / \mathrm{mL}(\alpha=0.18)$, SL form cylindrical micelles, as discussed earlier. Hereafter we try to elucidate the evolution of the cylindrical shape as a function of the degree of ionization at this same concentration. This analysis will provide valuable pieces of information concerning the overall nature of SL aggregates. The Porod region at high- $q$ values of the SANS spectra is of extreme importance because it allows to compare the overall surface-to-volume ratio $(\mathrm{S} / \mathrm{V})$ of the scattering objects independently from the value of the structure factor, $\mathrm{S}(\mathrm{q})\left(\lim _{q \rightarrow \infty} S(q)=1\right)$. This is done by plotting $\mathrm{Iq}^{4}$ as a function of the wavevector, $\mathrm{q}$. At high-q, the $\mathrm{Iq}^{4}$ value extrapolated at $\mathrm{q}=$ 
0, the Porod plateau, is directly proportional to S/V. Since, from simple geometrical considerations, $\mathrm{S} / \mathrm{V}$ is bigger for a sphere than for a cylinder, one can expect that, in a qualitative way, a cylindrical or wormlike micellar system have low $\operatorname{Iq}^{4}(q=0)$ values. Figure 6 represents the variation of $\mathrm{Iq}^{4}$ as a function of $\mathrm{q}$ for $\mathrm{C}=50 \mathrm{mg} / \mathrm{mL}$, for various degrees of ionization. In general, the Porod law is dependent on the volume fraction, $\phi$, which, in this case, is strictly equal for all samples allowing a straightforward comparison among the spectra (here, $\phi=5.2$ vol\%). At $\alpha=0.18$, micelles are known to be cylindrical objects whose length ranges between 130 and $160 \AA$ (aspect ratio around 5), as described after Figure 3; at higher ionization degrees, $\alpha=0.21$ and $\alpha=0.34$, the $\mathrm{Iq}^{4}$ curves display a slight increase at $\mathrm{q}=0$ but they are still very close to the one at $\alpha=0.18$. On the contrary, at $\alpha=0.80$, the $\mathrm{Iq}^{4}$ plateau, whose value $\left(\mathrm{Iq}^{4}=2.7 \cdot 10^{-4} \mathrm{~cm}^{-1} \cdot \AA^{-4}\right)$ is about $30 \%$ higher than the corresponding value for the $\alpha=0.18$ system $\left(\mathrm{Iq}^{4}=1.9 \cdot 10^{-4} \mathrm{~cm}^{-1} \AA^{-4}\right)$, suggests an increment in $\mathrm{S} / \mathrm{V}$ : the overall micellar surface increases. This phenomenon, in contrast with the expected evolution of SL micelles towards branched wormlike systems, can be interpreted with a shortening of the micellar length. Interestingly, after simple geometrical considerations, the relative gain in the calculated S/V for a rod-to-sphere transition is about 33\% and a similar behaviour can be found in the $\alpha$-driven cylinder-to-sphere transition in PS-PAA systems. ${ }^{30}$ The experimental representation of the structure factor, $S(q)$, calculated for the same data set (details can be found in Figure S3 and related discussion in the Supporting information) confirms the overall change of micellar shape going from $\alpha=0.18$ to $\alpha=0.80$.

As a general comment on the presence of a structure factor (one can also refer to scheme on repulsive interactions in Figure 2), one should retain that in many systems a minimum 5-10 wt\% surfactant concentration has to be reached in order to observe a prominent interaction peak, related to long-range repulsive interactions, but this value can increase up to $20 \mathrm{wt} \% .{ }^{31-38}$ Furthermore, in the specific case of alkylpolyglucosides (APG), no $\mathrm{pH}$-driven long-range ordering of micelles is found until concentration values reach at least $10-20 \mathrm{wt} \% .^{12,39-41}$ On the contrary, long-range intermicellar interactions for these systems can be controlled at lower concentrations by adding specific electrical charges at the micellar outer surface using ionic surfactants. ${ }^{42}$ In SL-based systems, electrical charges are autogenerated by $\mathrm{pH}$ (deprotonation of $\mathrm{COOH}$ group).

Local analysis using transmission electron microscopy under cryogenic conditions is performed on the $\alpha=0.80$ sample and shown in Figure 7a-b agree with the Porod plot analysis. The sample is constituted of spherical micelles, specifically indicated by the arrows 
in Figure 7b. The average cross-section radius $(\mathrm{Rm})$ and its size distribution are shown in Figure 7c and they correspond to $\mathrm{Rm}=21.9 \AA$ with $\sigma=2.2 \AA$. Larger micelles $(\mathrm{Rm}>60 \AA$ and L> $100 \AA$ ), which could actually explain the increasing I(q) in SANS at low-q in Figure 5, are also found in cryo-TEM images. Since their origin is not clear at the moment (do they form with time at $\alpha=0.8$ or are they already present at lower values?), we prefer to exclude them from the discussion.

Finally, combination of these techniques provides an answer to interpret the nature of the interaction peak observed in Figure 5 for $\alpha=0.80$. In regime 2, in close proximity to neutral $\mathrm{pH}$, SL micelles undergo a rod-to-sphere transition where long-range interactions, but not long-range order (at least not at $50 \mathrm{mg} / \mathrm{mL}$ ), appear.
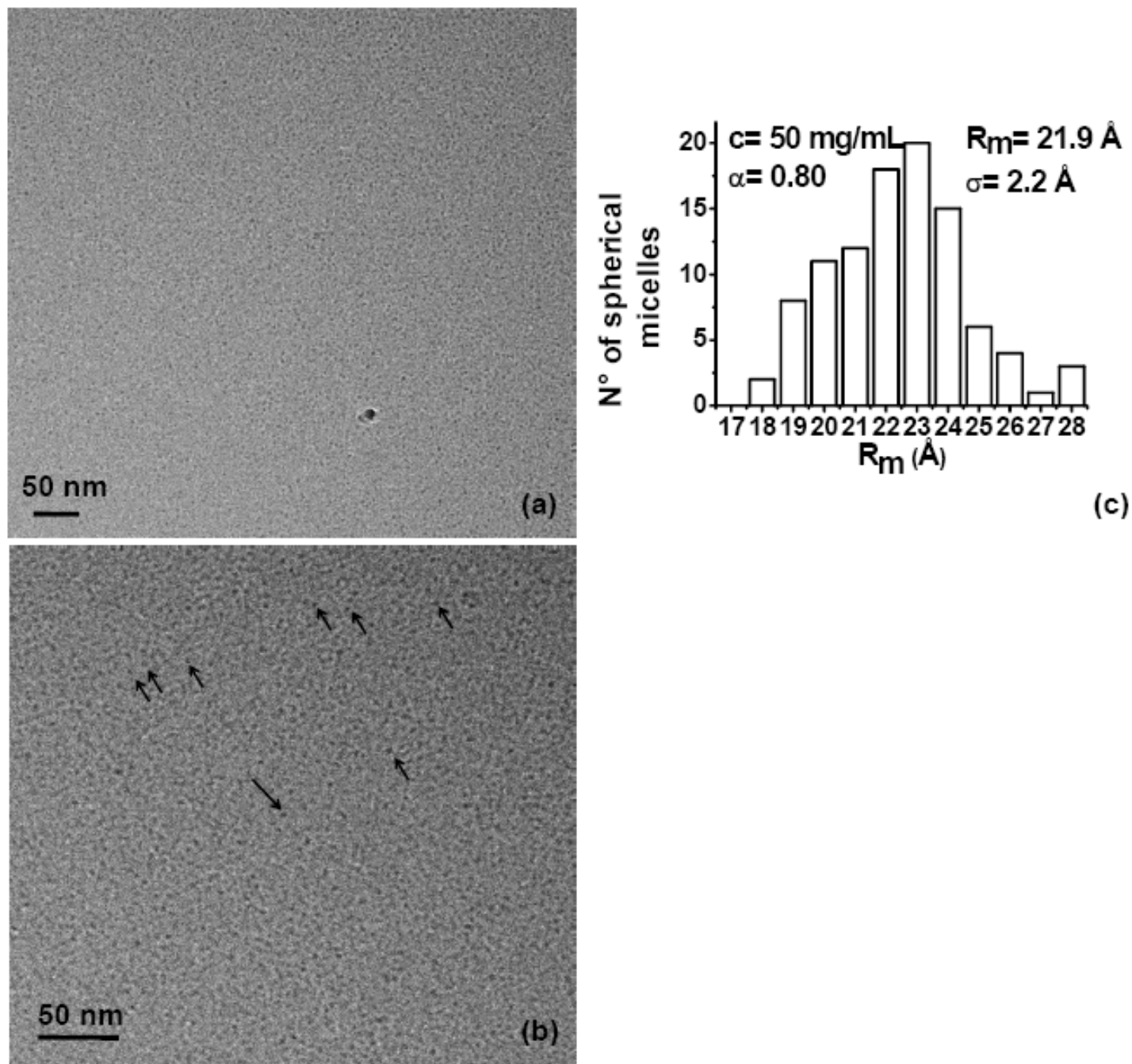

(c)

Figure 7 - (a-b) High resolution (magnification: $x$ 80k) cryo-TEM experiments performed on sample at $\mathrm{C}=50 \mathrm{mg} / \mathrm{mL}$ and $\alpha=\mathbf{0 . 8 0}$. The darker spherical objects represent the SL micelles frozen in a thin layer of amorphous ice. Images are corrected for the camera noise. (c) Size distribution of the cross-section micellar radius $\left(R_{m}\right)$ related to spherical micelles observed in (a-b). 
SL behaviour in Regime 3. At higher $\mathrm{COO}^{-}$concentration, the interaction peak in the mid- $q$ region is not detected anymore (Figure $4 b, \alpha=1$ ) while the scattered intensity at low- $q$ increases without reaching a plateau. Figure 8 focuses on the SANS experiments performed on two samples at $\mathrm{C}=5 \mathrm{mg} / \mathrm{mL}$, both at $\alpha=1$, but at different $\mathrm{NaOH}$ concentration. First of all, spectra can be superimposed in the whole q-range indicating no apparent influence of the amount of sodium hydroxide at $\alpha=1$. The discussion next will be focused on the system at $[\mathrm{NaOH}]=12.5 \mathrm{mM}$, corresponding to $\mathrm{pH}=11$. Please remark that a slight excess of base with respect to the full ionized $\mathrm{COO}^{-}$might be required to achieve this specific regime.

Secondly, the signal-to-noise ratio of $\mathrm{I}(\mathrm{q})$ throughout the whole q-range is poor, if compared to the same system $(\mathrm{C}=5 \mathrm{mg} / \mathrm{mL})$ at $\alpha=0.31$ (Figure $4 \mathrm{~b}$ ), meaning that the lower concentration value is not responsible for such loss in signal intensity. Due to the poor quality of SANS data, we prefer not to pursue a detailed discussion based on the classical analysis of the $\log [\mathrm{I}(\mathrm{q})]$ vs. $\log [\mathrm{q}]$ slopes. A fit of the high-q region using a sphere form factor provides a gross estimation of the micellar cross-section $(\mathrm{R}=16 \AA)$, as shown in Figure $\mathrm{S} 2$ in the Supporting information.

On the contrary, the very strong increase in I(q) at low q-values $\left(8.38 \cdot 10^{-3} \AA^{-1}<\mathrm{q}<\right.$ $\left.7.32 \cdot 10^{-2} \AA^{-1}\right)$, confirmed by additional experiments at very low-q (q<8.38 $10^{-3} \AA^{-1}$ ) for the $[\mathrm{NaOH}]=12.5 \mathrm{mM}$ system (full circles in Figure 8a), give a strong hint of the existence of large micellar $(\mathrm{R}>50 \mathrm{~nm}$ ) structures whose size and shape cannot be determined by SANS (for a better understanding, one can refer to the schematic representation of fractal geometries in Figure 2). The diffuse q behaviour and very low intensity above $0.02 \AA^{-1}$, which could indicate unstable micellar objects and interfaces, is more difficult to explain. Similar datawere reported by Zhang et al., ${ }^{39}$ who also experienced diffuse high- $q$ scattering in alkylglucoside solutions and attributed it to large fluctuations in the position of molecules within the micelles which depends on the less marked hydrophilic/hydrophobic character of these surfactants. In a slightly different system, Bendejacq et al. ${ }^{30}$ have shown a swelling effect of $\mathrm{COOH}$ brushes in PS-PAA copolymers at increasing $\alpha$. Here, it can be symptomatic of both a less stable interface and a reduction in the amount of small-sized micelles, otherwise abundant at lower $\alpha$-values. In other words, as discussed above for regime 2 , the lower the signal at high-q, the lower the $\mathrm{S} / \mathrm{V}$. 


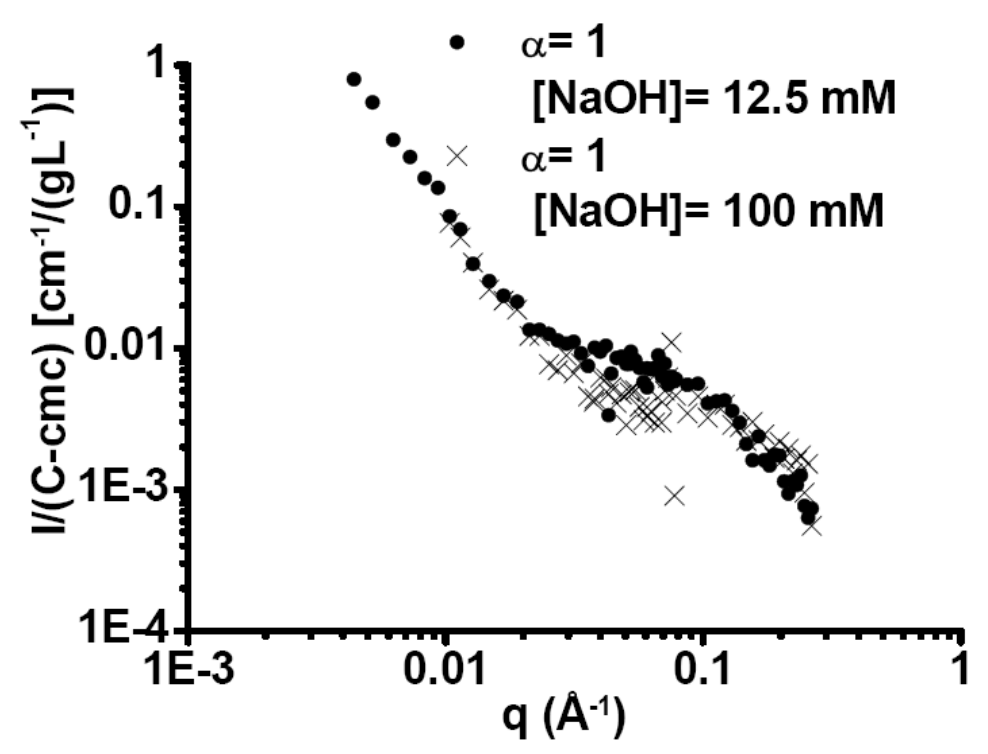

Figure 8 -SANS experiments performed on two SL solutions at $\alpha=1$ but different NaOH concentrations. Data on the $[\mathrm{NaOH}]=12.5 \mathrm{mM}(\mathrm{pH}=11)$ system (full circles) were completed with ultra-low angle experiments $\left(q<8.38 \cdot 10^{-3} \AA^{-1}\right)$ to confirm the increase of $I(q)$.

To complete SANS experiments and better explain the nature of the aggregates, we performed Transmission Electron Microscopy under cryogenic conditions on the sample at $\alpha=1$ and $[\mathrm{NaOH}]=12.5 \mathrm{mM}$, (Figure 9a-d). First of all, the amount of individual micelles seems to be far less important with respect to systems studied in Figure 7; most attempts to observe them inside the amorphous ice holes were unfruitful but the low-intense signal at high-q in SANS, as shown by the fits in Figure S2 in the Supporting information, cannot completely exclude their presence. On the contrary, we could observe separate regions composed of a dense system of elongated, branched, aggregates, as shown in the overview image in Figure 9a. A close look at the micellar region shows an ill-defined system composed of interconnected tubules whose average side-diameter is about $13 \pm 3 \mathrm{~nm}$ (see arrows in Figure 9b). The overall dimensions of the clusters are comprised between 100 and $500 \mathrm{~nm}$ and the unorganized network identifies hollow cavities whose size ranges between few nanometers to $100 \mathrm{~nm}$. Individual branches of much smaller size or long (>100 nm) flexible objects are also observed (Figure 9 b-c). The exact description of the supramolecular aggregates formed under high $\mathrm{pH}$ conditions reveals to be a hard task as well as the explication of their origin. We could not easily find in literature a similar surfactant system with such a different behaviour as a function of $\mathrm{pH}$. Nevertheless, several synthetic asymmetric bolaform surfactants have been reported to form nanotubes, generally referred to as lipid nanotubes. ${ }^{43}$ In these works, individual nanotubes were clearly identified and random 
aggregation, similar to what it is observed here, does not occur as much. For one particular surfactant, whose molecular structure is close to the one of sophorolipids, well-defined nanotubes are obtained under acidic conditions but more globular, ill-defined, structures were reported at higher $\mathrm{pH}$ values. ${ }^{26}$ Unfortunately, the authors did not further investigate the influence of high $\mathrm{pH}$ on the supramolecular assembly of their compounds. In the SL-based system obtained under basic conditions we could seldom observe isolated elongated aggregates and in any case they cannot be closely related to well-defined nanotubular objects. On the contrary, as one can observe in Figure 9b (black circles), where these structures could be isolated, they are composed of an elongated tubular scaffold with local hemispherical, vesicle-like, protuberances. An interesting tubular-to-vesicle transition as a function of temperature was described by Imae at $a l .{ }^{44}$ during the study of a fluorinated-hydrogenated glucophospholipid. They attributed such a phenomenon to a local molecular variation of the surfactant packing. The average side-diameter of our tubules is comparable with the one reported by several authors on lipidic nanotubes. ${ }^{26,44}$ In particular, Masuda and Shimizu ${ }^{26}$ were able to correlate the stacking periodicity and the size of the inner tube diameter with the chain length and packing of their bolaamphiphiles. In our system, sophorolipids have a C18 chain, which, according to the model proposed by Masuda and Shimizu and in the case that it could be applied to this specific system, should provide tubules whose diameter ranges between 18 and $20 \mathrm{~nm}$. The average side-diameter observed here (13 $\pm 3 \mathrm{~nm})$ is slightly smaller but one should probably consider the influence of the SL C=C double bond on the local molecular packing. 

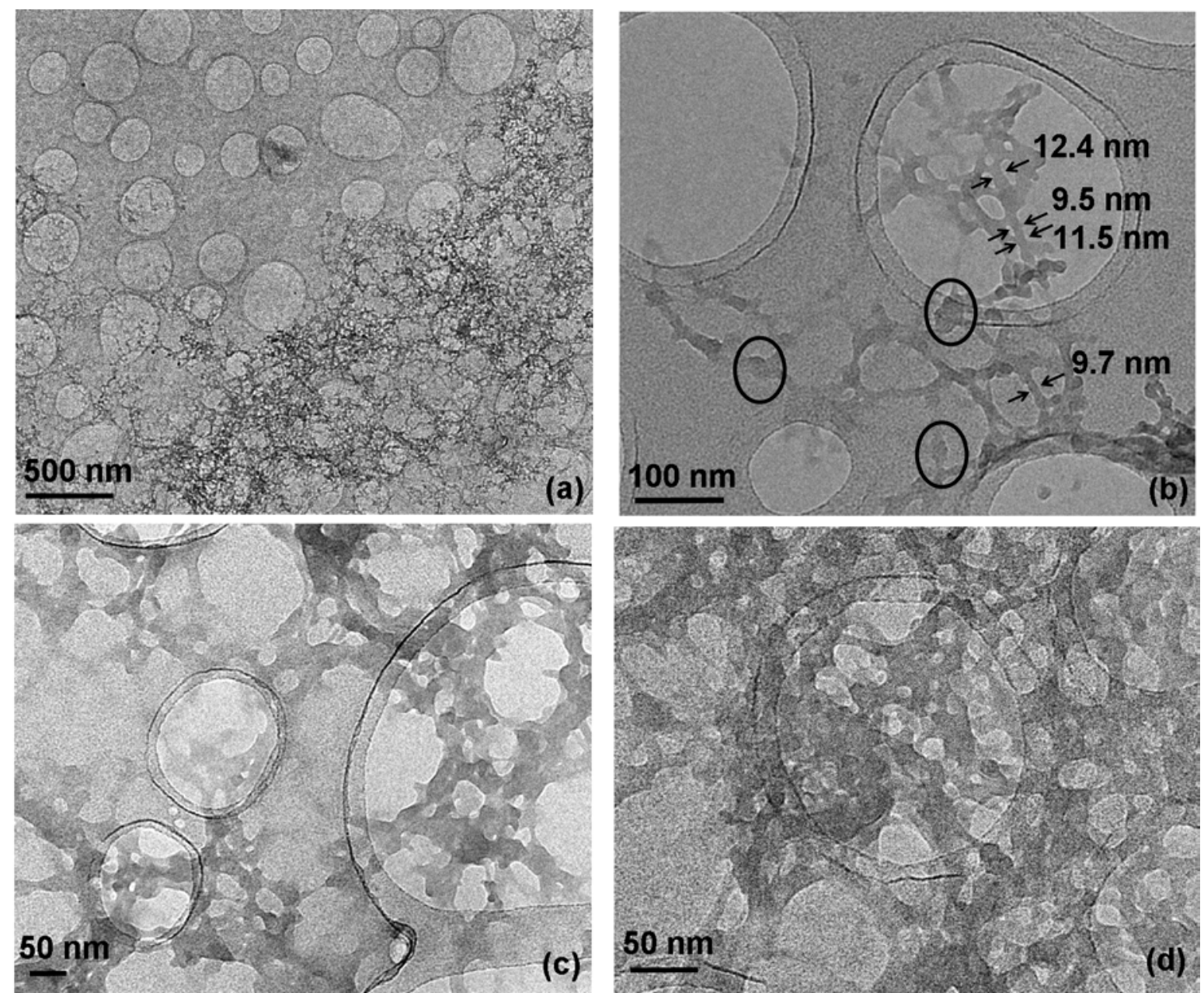

Figure 9 - Cryo-TEM images performed on the SL solution at $\alpha=1$ and $[\mathrm{NaOH}]=12.5 \mathrm{mM}$. Note that the grey homogeneous background is the classical amorphous holey carbon layer on top of the TEM copper grid while the circular shapes indicate the amorphous-ice covered holes within the membrane.

SL molecular configuration. The degree of ionization introduces negative charges determining, at mild $\alpha$ values, electrostatic repulsive forces with a strong influence on both the shape and size of the aggregates. An important issue is the local conformation of the SL molecule and, in particular, the localization of the $\mathrm{COOH} / \mathrm{COO}^{-}$group within the micelle as a function of $\alpha$. First of all, upon neutralization, one can also reasonably suppose that $\mathrm{COO}^{-}$ groups move towards the external micellar surface, being responsible for their negative charge. Zeta-potential, $\zeta$, experiments are useful to determine in a qualitative way the charge at the micellar surface. For instance, at $\mathrm{C}=5 \mathrm{mg} / \mathrm{mL}$, the following values for zeta-potential, $\zeta=-29.7 \mathrm{mV}$ and $\zeta=-49.1 \mathrm{mV}$, were measured respectively for the $\alpha=0.31$ and $\alpha=0.62$ systems while at $\mathrm{C}=50 \mathrm{mg} / \mathrm{mL}, \zeta$ varies between $-10.0 \mathrm{mV}$ and $-25.0 \mathrm{mV}$ for $0.2<\alpha<0.8$. As expected, in all cases the micelles show a negative surface charge, whose absolute value seems to increase with $\alpha$ at a given concentration. Local electrostatic repulsions between 
$\mathrm{COO}^{-}$groups may also account for the increase in the micellar curvature at $\mathrm{C}=50 \mathrm{mg} / \mathrm{mL}$, going from cylinders to spheroids when $\alpha$ shifts from 0.18 to 0.80 , as discussed earlier.

To draw a picture of the local molecular conformation of SL within the micelles, we run a set of ${ }^{1} \mathrm{H}-{ }^{1} \mathrm{H}$ two-dimensional Nuclear Overhauser Enhancement Spectroscopy (2D NOESY) NMR experiments on three SL solutions at variable $\alpha$-values, each corresponding to a specific regime. 2D NOESY NMR is an effective method to study the three-dimensional structure of large molecules that have long motional correlation time, such as proteins. ${ }^{45,46}$ This type of 2D spectrum displays NOE cross-peaks located at the chemical shift of protons that are correlated through magnetization transfer mediated by dipole-dipole interactions. The effect occurs through space and is independent of covalent bonds. It is strongly distancedependent and the protons normally need to be separated by less than $5 \AA$ to be observed in NOESY spectra. Cross-signals in a NOESY spectrum rely on the cross-relaxation of longitudinal magnetization during a selected mixing time. 2D NOESY is currently employed to characterize internuclear distances and molecular conformation in surfactants micellar systems $^{47,48}$ and it will be employed here to probe proton-proton proximities within SL; in particular, we will focus on the interactions involving the $\mathrm{COOH}$ group but one should recall that it is not possible to distinguish between intramolecular and intermolecular interactions.

${ }^{1} \mathrm{H}-{ }^{1} \mathrm{H}$ 2D NOESY experiments (Figure $11 \mathrm{a}, \mathrm{b}$ ) were run on solutions at $\alpha=0.18, \alpha=$ $0.80(\mathrm{C}=50 \mathrm{mg} / \mathrm{mL})$ and at $\alpha=1(\mathrm{C}=5 \mathrm{mg} / \mathrm{mL} ;[\mathrm{NaOH}]=12.5 \mathrm{mM})$ while mixing time, $\tau_{\mathrm{m}}=$ $300 \mathrm{~ms}$, was selected on the basis of a build-up curve run for $50 \mathrm{~ms}<\tau_{\mathrm{m}}<900 \mathrm{~ms}$, typical values used in the study of surfactant-based systems in water above their cmc value (the acidic SL has $\mathrm{cmc}_{\mathrm{SL}-\mathrm{COOH}}=0.11 \mathrm{mg} / \mathrm{mL}$ while its $\mathrm{cmc}$ after deprotonation is cmc $\mathrm{CL}-\mathrm{COO}=0.15$ $\mathrm{mg} / \mathrm{mL}$. Please refer to sample preparation section and Figure S4 in Supporting information for more details on cmc data of SL). The ${ }^{1} \mathrm{H}$ chemical shift attribution is done on the basis of the 1D spectrum (top of the 2D NOESY maps and scheme in Figure 11c): ${ }^{17} 1$ ) oleic acid moiety: $\mathrm{H}_{2}\left(\mathrm{HOOC}-\underline{\mathrm{C}}_{2}-\mathrm{CH}_{2}-\right)$ at $2.15 \mathrm{ppm} ; \mathrm{H}_{3}\left(\mathrm{HOOC}-\mathrm{CH}_{2}-\underline{\mathrm{C}}_{2}-\right)$ at $1.51 \mathrm{ppm} ; \mathrm{H}_{9,10}$ ($\underline{\mathrm{HC}}=\mathrm{C} \underline{\mathrm{H}}-)$ at $5.30 \mathrm{ppm}$. 2) sophorose moiety: $\mathrm{H}_{1^{\prime}}, \mathrm{H}_{1^{\prime \prime}}$ (anomeric $\left.-\mathrm{O}-\mathrm{C} \underline{\mathrm{H}}-\mathrm{O}-\right)$ at $4.45 \mathrm{ppm} ; \mathrm{H}_{(2-}$ 6)', $\mathrm{H}_{(2-6) "}(-\mathrm{O}-\mathrm{C} \underline{\mathrm{H}}-)$ at 3.16-3.8 ppm.

The $\mathrm{H}_{3}$ signal, in close proximity to the carboxylic acid, is a good for interactions between the end of the oleic acid (OA) chain and the sophorose headgroup. The NOESY response of the sample at $\alpha=0.18$ (Figure 11a) shows several cross-peaks, highlighted in grey in the F1-dimension and connected through the black lines, related to the $\mathrm{H}_{3}$ carbon signal. First of all, dotted lines identify close-neighbours connections between $\mathrm{H}_{3}$ with the oleic acid 
backbone $(\delta=1.26 \mathrm{ppm})$ and $\mathrm{H}_{2}$ while solid lines identify several interesting long-range connections with $\mathrm{H}_{9,10}$ and sophorolipid head $\left(\mathrm{H}_{1}\right.$, $\mathrm{H}_{1 "}, \mathrm{H}_{(2-6)}$, $\left.\mathrm{H}_{(2-6)}\right)$. For comparison, at $\alpha=$ 0.80 and at equal mixing time (Figure 11b), short-range interactions like $\mathrm{H}_{3}-\mathrm{H}_{2}$ are still detected, as expected, while the long-range connections with sophorose are almost completely lost. At $\alpha=1$ (map not shown), no cross-peaks at all are detected, except for those relating to close neighbours interactions. In Figure 11d, we compare, as a function of $\alpha$, the slices extracted from the 2D NOESY map at $\tau_{\mathrm{m}}=300 \mathrm{~ms}$, corresponding to the $\mathrm{H}_{3}$ resonance $(\delta=$ $1.51 \mathrm{ppm}$; refer to the solid arrow on the figure): the intensity of the corresponding $\mathrm{H}_{3}$ sophorose cross-peaks decreases with $\alpha$. A similar trend was observed at lower mixing times ( $\tau_{\mathrm{m}}=50 \mathrm{~ms}$ ), at which ${ }^{1} \mathrm{H}-{ }^{1} \mathrm{H}$ spin diffusion artefacts may considered to be minimized.

NOESY experiments qualitatively show that the $\mathrm{COOH}$ environment of oleic acid backbone is in close interaction with the sophorose moiety at low $\alpha$ while this is not the case at higher ionization degrees. Strong hydrogen bonds between $\mathrm{COOH}$ and $\mathrm{OH}$ groups in sophorose may explain this behaviour even if it is impossible to discriminate between interactions occurring within the same or between two adjacent SL molecules. In the field of bolaform surfactants having an all-trans conformation of the aliphatic chain, adjacent molecules are proposed to be either in a parallel or an anti-parallel configuration, ${ }^{26,49}$ as also shown in Figure 10. In this case, NOESY experiments could help probing interactions in close spatial environments and one could probably discriminate between them. Unfortunately, if folding of the alkyl chain occurs, as it could be the case for sophorolipids (one should not forget that SL are mostly produced in their close lactone form and are characterized by a cis$\mathrm{C}=\mathrm{C}$ double bond), clear-cut discrimination through NOESY NMR experiments becomes highly challenging, if not impossible. In this case, one would expect the same spatial proximities in both the parallel and anti-parallel configurations, as sketched by the pointed lines in Figure 10 (cis configuration). 


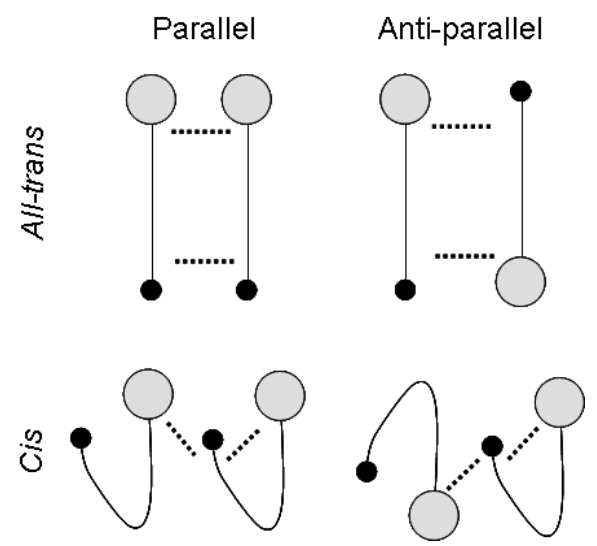

Figure 10 - Scheme of the possible parallel and anti-parallel configurations of bolaform surfactants in case of an all-trans configuration of the alkyl chain, as propoised in ref. 26, and in presence of a cis $\mathrm{C}=\mathrm{C}$ double bond, as it occurs in sophorolipids. Pointed lines represent close-rage through-space interactions.

In the $\alpha=1$ system, one can observe the absence of long-range intermolecular interactions, which is partly unexpected even if that confirms the absence of small micellar objects. Yet, it is not excluded that the diffusion dynamics of the large aggregates observed in cryo-TEM are too slow and their transverse relaxation time too small to be observed under the actual conditions; the signal for this particular system may actually corresponds to free SL molecules. Further experiments are ongoing to verify this hypothesis. To account for these observations one can formulate the hypothesis that the large micellar aggregates contain mobile SL molecules, probably forming a mono-molecular layer, which are solvated with large amount of water, both inside and outside the tubular structures, implicitly suggesting a hollow tubular configuration, as often reported for related systems. ${ }^{26,50}$ 

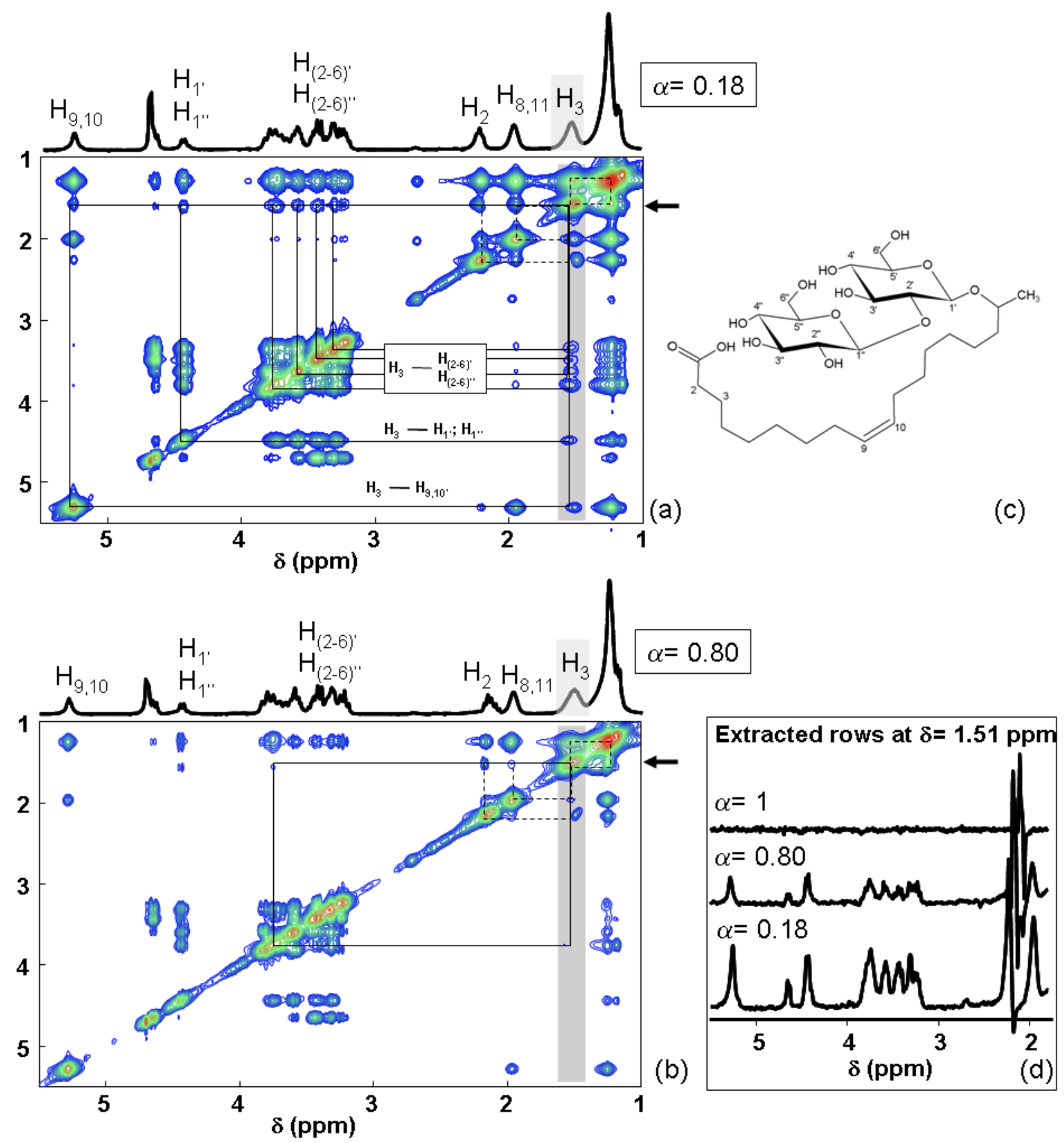

Figure $11-{ }^{1} \mathrm{H}-{ }^{1} \mathrm{H}$ 2D NOESY NMR experiments performed at mixing time of $300 \mathrm{~ms}$ for SL solution at $\mathrm{C}=50 \mathrm{mg} / \mathrm{mL}$ at (a) $\alpha=\mathbf{0 . 1 8}$ and (b) $\alpha=\mathbf{0 . 8 0}$. (c) SL molecule with typical convention for ${ }^{1} \mathrm{H}$ chemical shift assignment. (d) Comparison of extracted rows at $\delta_{1 \mathrm{H}}=1.51 \mathrm{ppm}$ (black arrow) from the corresponding 2D

NOESY experiments. We remind that both samples are prepared at the same concentration and 2D NOESY spectra are acquired using the same NMR parameters and, most importantly, post-acquisition treatment (e.g., contour plot height).

The molecular structure of SL resembles the one of glycolipids but $\mathrm{COOH}$ group has an important impact on the self-assembly process and the stability of micelles as a function of $\mathrm{pH}$ and time. Unfortunately, most studies on natural surfactants were done on classical glycolipids and no specific term of comparison involving SL is available at the moment. The theoretical micellar radius of a fully elongated SL molecule, corresponding to a $\beta$-anomer 
conformation, should include the contribution of oleic acid (OA) (approximately $22 \AA^{51,52}$ ) and sophorose, whose size can be estimated to be below $10 \AA$. This value is obtained by comparison with $N$-dodecyl- $\beta$-maltoside $\left(\mathrm{C}_{12} \mathrm{G}_{2}\right)$, in which, according to experimental and modelled data, maltose (a common disaccharide comparable to sophorose) does not contribute to more than $6 \AA$ to the size of $\mathrm{C}_{12} \mathrm{G}_{2}$ in its linear conformation ( $\beta$-anomer). ${ }^{53}$

Under these conditions, one expects a cross-sectional radius for SL micelles of approximately $30 \AA$. In reality, the experimental data measured by SANS are $30 \%$ to 50\% lower depending on the micellar shape (sphere, ellipsoid or cylinder) in the low ionization degree regime, as discussed before and shown in Table 1. These discrepancies are too large to be neglected. Typical cross-sectional radius reported in litterature for sodium oleate micelles is $26 \AA^{54}$ while for an oleic-acid based glycolipid, whose polar head is constituted by a disaccharide and referred to as $\mathrm{OM},{ }^{40,55}$ is $25 \AA$. It is very interesting to note that almost no discrepancy exists between the cross-section sizes of sodium oleate and OM, despite the additional disaccharide group in the last compound. As shown by Abel et al. ${ }^{53}$ for $\mathrm{C}_{12} \mathrm{G}_{2}$, this can strongly depend on the $\alpha$ (right angle bending between the sugar and tail) or $\beta$ anomeric configuration of the disaccharide with respect to the aliphatic chain. The same authors have also outlined the importance of the strong hydrogen bonding in which the maltose head is involved and that $10 \%$ of the hydrophobic chain is permeable to water. The cross-sectional radius measured by SANS for SL spherical micelles (5 mg/mL, $\alpha=0.31$ ) is $26.1 \AA \AA$, which suggests that the sophorose group acquires a $\alpha$-anomer conformation. Nevertheless, this is in contrast with previous works in which $\beta$-conformation was reported for SL. ${ }^{17,56}$ As a first conclusion, one can then say that the OA moiety does not acquire a fully elongated conformation. When a sphere-to-cylinder transition occurs or when an ellipsoid form factor is used, the cross-sectional radius determined by SANS is even smaller, that is about $15 \AA$. Cross-sections for cylindrical micelles are reported to be equal or even slightly smaller (< $10 \%$ ) than the corresponding spherical systems in many systems ranging from classical surfactants like $\mathrm{CTAB}^{33}$ to glucose based compounds. ${ }^{39,40}$ Nevertheless, a $40 \%$ difference as observed in SL is too large to be explained by chain conformation, packing or curvature differences, as usually done.

NOESY experiments above may provide an explanation to these discrepancies. The folding of the OA moiety, which was described before for SL, ${ }^{56}$ can be driven by hydrogenbond interactions between the $\mathrm{COOH}$ group and the sophorose, either within the same or, most likely, between two adjacent SL molecules. In this case, the effective length of the SL 
molecule and, consequently, of the micellar radius is smaller, especially if sophorolipids molecules are parallel to each other and the oleic acid backbone is folded, as shown in Figure 10 (cis configuration). Nevertheless, one should also consider the possibility of an antiparallel configuration (Figure 10), as also proposed in ref. 26 for bolaform surfactants and discussed above. Further experiments and structural modelling are needed to confirm this assumption. At a higher ionization degree, the $\mathrm{COO}^{-}$has weaker interactions with sophorose and its hydration degree is probably higher, which may induce a non-negligible water intrusion at the micelle/solvent palisade and, eventually, within the micellar core. Under these conditions we expect an increase in the micellar radius, which seems to be confirmed by cryoTEM analysis in Figure 7 indicating an average $21.9 \AA$ radius for the $50 \mathrm{mg} / \mathrm{mL}$ system at $\alpha=$ 0.80. At even higher $\alpha$, the overall ionization effect on the structure of SL and the corresponding effect on the overall aggregate size, morphology and cross-section is not very clear, yet. Larger micellar aggregates are identified by the combination of SANS and cryoTEM but the lack of any cross-peaks in 2D NOESY NMR experiments could reveal a strong SL mobility and an unstable micellar interface, probably integrating bigger amounts of water within the large tubules shown by cryo-TEM experiments. ${ }^{1} \mathrm{H}$ 1D NMR experiments (results not shown) may corroborate this hypothesis: the ${ }^{1} \mathrm{H}$ NMR signal corresponding to the $\mathrm{H}_{2}$ group of SL for $\alpha=1$ is systematically narrower than at lower $\alpha$ and its chemical shift is slightly deshielded. This last feature is also observed for free oleate molecules below their critical micelle concentration. ${ }^{57}$ In any case, under these conditions, the anti-parallel arrangement of two adjacent SL molecules is a hypothesis to be considered with good probability where the free $\mathrm{COO}^{-}$group would be solvated by water both inside and outside the tubule. Whatever the configuration adopted by adjacent SL molecules, the micellar surface becomes negatively charged with $\mathrm{pH}$.

Overview on SL conformation and self-assembly. Scheme 1 provides a schematic overview over the possible $\alpha$-driven micellar regimes of SL in water described above. Please note that adjacent SL molecules are presented in a parallel configuration but one should not exclude, especially at high ionization degree, the possibility of an anti-parallel form, as proposed elsewhere, ${ }^{26}$ and discussed above.

- low $\alpha$ : micelles form above the cmc and their shape evolves from spherical to wormlike (curvature decreases). The micellar surface is mostly neutral and excluded volume drives intermicellar interactions, in which electrostatic effects do not play a major role. The behaviour of SL in this regime is similar to classical glycolipid-based surfactants. Locally, the 
$\mathrm{COOH}$ groups seem to interact through hydrogen bonding with the sophorose moiety. Entanglement of adjacent oleic acid chains cannot be excluded. Such a hydrogen bonding configuration is explicitly shown in Scheme 1.

- Intermediate $\alpha$. deprotonation of $\mathrm{COOH}$ into $\mathrm{COO}^{-}$introduces electrical charges located at the surface of the micellar objects. Micellar shape is strongly affected since its curvature increases: at a given concentration, a rod-to-sphere transition is observed and a mixture of spherical and obloidal micelles is largely observed. The introduction of electrostatic intermicellar repulsive effects result in long-range interactions at concentration values as low as $50 \mathrm{mg} / \mathrm{mL}$. The effect of concentration at intermediate $\alpha$ was not studied here.

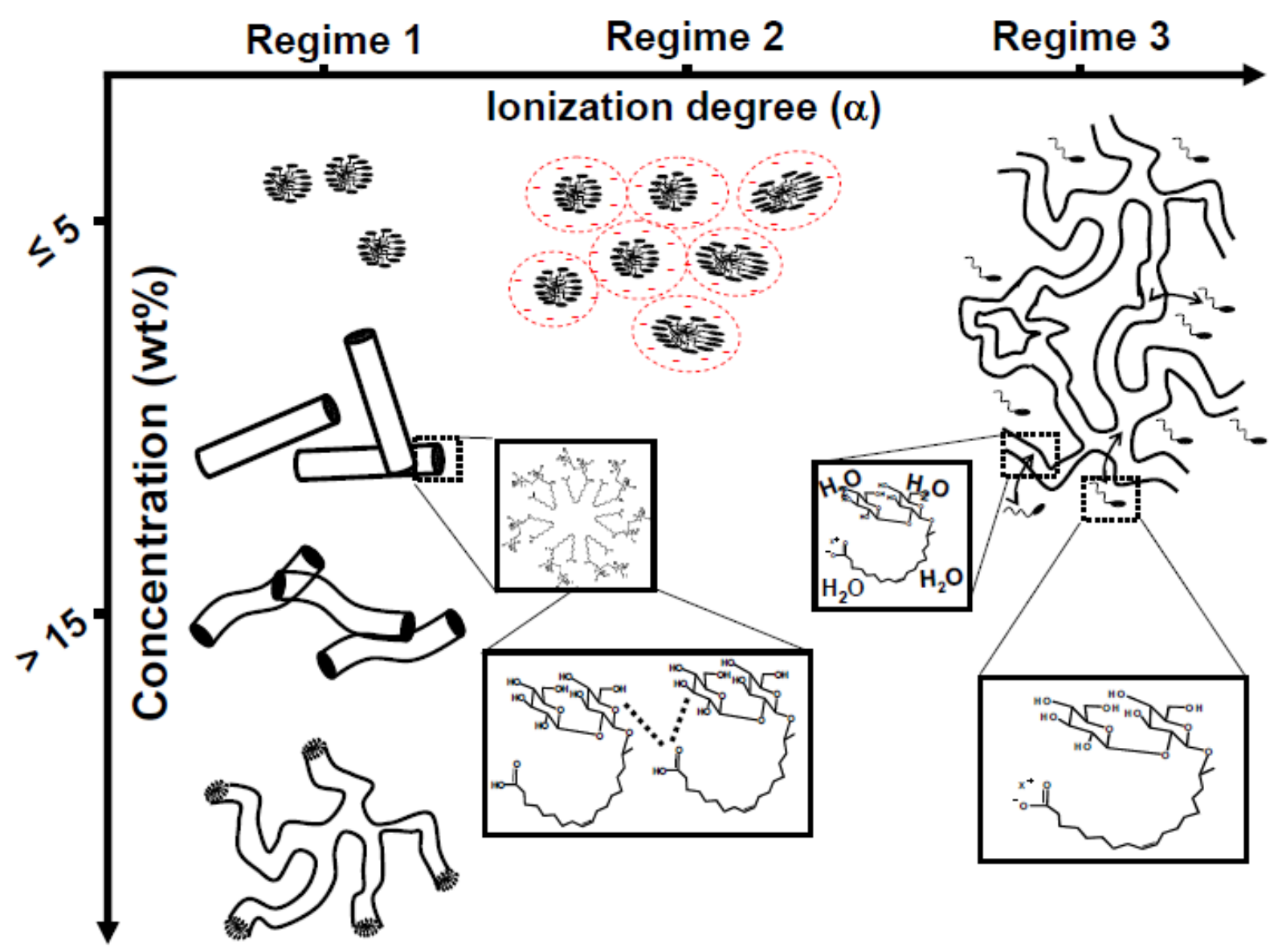

Scheme 1 - Schematic overview of SL self-assembly in $\mathrm{D}_{2} \mathrm{O}$ as a function of concentration and ionization degree within $24 \mathrm{~h}$ from the preparation of the solution. Please note that drawings are not scaled.

- $\alpha=1$ : when all $\mathrm{COOH}$ turn into $\mathrm{COO}^{-}$and in probable excess of added base, the shape and size of micelles is drastically affected: very long (>100 nm) tubules (diameter $>10 \mathrm{~nm}$ ) entangle together to form micellar aggregates of ill-defined shape and surrounding water-rich gaps. The amount of free micelles is largely reduced according to high-q SANS and cryo- 
TEM arguments while solvation of SL molecules by water within the large aggregates is not excluded. The hydrophilic/hydrophobic balance of SL is drastically reduced causing a higher SL solubility and rapid disruption of the micelle/water interface. The exact nature and composition of these aggregates and the local conformation of SL in this regime needs further studies to be better understood.

\section{Conclusion}

In this work we have investigated the self-assembly properties of sophorolipids (SL), a bioderived glycolipid containing a free $\mathrm{COOH}$ group, as a function of the degree of ionization of its internal $\mathrm{COOH}$ group. SL are biosurfactants with a very low cmc and which form, under acidic conditions, spherical, cylindrical and wormlike micelles as a function of concentration. Above $300 \mathrm{mg} / \mathrm{mL}$, the micellar entanglement strongly increases but SANS experiments show that long-range interactions are not directed by electrostatic repulsive forces, as it usually occurs for ionic surfactants, but by the higher volume fraction occupied by the compound. This behaviour, which is identified in this study as regime 1 , is very close to many other sugar-derived surfactants and it depends on the non-ionic nature of these compounds. On the contrary, slight increase in $\mathrm{pH}$ induces the ionization of the $\mathrm{COOH}$ group, which is typical for SL, and the consequent increase in the ionization degree, $\alpha$. At $\alpha<1$, the nature of intermicellar interactions drastically change and regime 2 starts: two phenomena occur at the same time: 1) micelles undergo a low-to-high curvature transition (cylinder-tosphere/ellipsoid) due to the introduction of negative charges at their surface; 2) electrostatic repulsive forces drive long-range interactions between micellar objects, as observed both by SANS and nicely confirmed by cryo-TEM microcopy. Interestingly, SL behaviour in regime 1 and 2 clearly indicates that one can tune, for a given shape, the properties of micellar surface, from neutral to negatively charged, making these objects very versatile for a number of applications in nanoscience related fields. At $\alpha=1(5 \mathrm{mg} / \mathrm{mL})$ a new, unexpected, state of SL supramolecular assemblies occurs. In regime 3, all SL molecules are negatively charged and their hydrophilic/hydrophobic character is less pronounced. The micelle/water interface seems to be disrupted (SANS behaviour at high-q) and the presence of monomers is now favoured (as also seen by ${ }^{1} \mathrm{H}$ NMR); nevertheless, both SANS and DLS experiments agree on the existence of very long (> $100 \mathrm{~nm}$ ) micellar tubular (average diameter is $13 \mathrm{~nm}$ ) aggregates organized in a net-like structure of ill-defined shape, as shown by cryo-TEM experiments. The true SL behaviour in this regime needs still to be clarified. At a local scale, NOESY experiments suggest that intra/intermolecular $\mathrm{COOH}$-sophorose hydrogen bonding 
characterizes the low- $\alpha$ regime but they do not bring any clarification on the local SL parallel or anti-parallel configuration.

These experiments show that sophorolipids constitute an extremely versatile micellar system whose $\mathrm{pH}$-driven properties make them an interesting stimuli-responsive compound at very low concentration values ( $<5 \mathrm{wt} \%$ ), which is quite atypical for comparable surfactant-based systems. The three different regimes, neutral and charged micelles at low-medium degree of ionization followed by large net-like aggregates at high $\alpha$ clearly show the potential versatility of these molecules.

\section{Experimental}

Synthesis of SL. Sophorolipids were produced by Candida bombicola (teleomorph: Starmerella bombicola) ATCC 22214. The yeast was cultivated on medium as described before. ${ }^{58}$ A fed-batch fermentation was run in a Biostat ${ }^{\circledR}$ B culture vessel (Sartorius-BBI Systems) with a maximum working volume of $1.5 \mathrm{~L}$. Temperature $\left(30^{\circ} \mathrm{C}\right), \mathrm{pH}(3.5)$, stirring rate $(800 \mathrm{rpm})$ and airflow rate $(1 \mathrm{vvm})$ were controlled by the Biostat ${ }^{\circledR}$ B control unit. 100 $\mathrm{mL}$ of an overnight grown shake flask culture was used to inoculate the fermentor. For maintaining $\mathrm{pH}, 5 \mathrm{M} \mathrm{NaOH}$ was used. There was no correction for a too alkaline $\mathrm{pH}$ and fermentation started at $\mathrm{pH} 5.8$ and was allowed to drop spontaneously till 3.5. Later unalterable incensement was seen as the end of the fermentation process. Feeding of rapeseed oil (Sigma) was started 48 hours after inoculation, and was adjusted to the consumption rate. Additional glucose (50 g/L) was added 150 hours after inoculation. Sophorolipids were extracted by the following procedure: 3 volumes of ethanol were added to the fermentation medium and yeast cells were removed by centrifugation. The water-ethanol mixture of the supernatants was removed under reduced pressure in a rotavapor. 2 volumes of ethanol were added to dissolve the sophorolipids and the residual hydrophobic carbon source. The mixture was passed over a Whatman filter to remove the water soluble components and ethanol was evaporated under reduced pressure in a rotavapor. Solid substances were dissolved in water (pH 6.5) and residual oil and fatty acid were extracted by an equal volume of hexane. The sophorolipid mixture (about $80 \% \mathrm{C} 18: 1$ ) is comparable to what it was reported by other authors. ${ }^{59}$ Finally, hydrolysis of the mixture to obtain the acidic $\mathrm{COOH}$ form of SL is performed with $5 \mathrm{M} \mathrm{NaOH}$ solution and extracted with pentanol according to procedure described in Ref. 60. The product is then further purified on a chromatographic column using silica gel 60 as stationary phase and a 80:20 dichloromethane:methanol mixture. Thin Layer Chromatography was performed on silica gel 60 plates (Whatman) using the same solvent 
mixture. Staining is done with phosphomolybdic acid (5 g in $50 \mathrm{~mL}$ ethanol solution) and revelation is performed by warming up the substrate. Analysis is done with ${ }^{1} \mathrm{H}$ and ${ }^{13} \mathrm{C}$ NMR $\left(\mathrm{CDCl}_{3}: \mathrm{CH}_{3} \mathrm{OD}=1: 1\right)$. An average downfield $5 \mathrm{ppm}$ shift from $172 \mathrm{ppm}$ to $177 \mathrm{ppm}$ is used as reference to verify the ester hydrolysis of the $\mathrm{COOH}$ group. The final compound is mainly (> 90\%) constituted by acidic sophorolipids, as also verified by ${ }^{1} \mathrm{H}$ NMR (results not shown).

Sample preparation. Different amounts of SL are added to $\mathrm{D}_{2} \mathrm{O}$ (Aldrich) solutions at concentration, $\mathrm{c}=5,50,300,400$ and $500 \mathrm{mg} / \mathrm{mL}$ corresponding to volume fraction, $\Phi=$ 0.52\%, 5.20\%, 31.25\%, 41.6\%, 52.08\%. pH was increased using $\mu$ molar amounts of $0.5 \mathrm{M}$ and $1 \mathrm{M} \mathrm{NaOH}$ solutions, which were prepared in $\mathrm{D}_{2} \mathrm{O}$, in order to limit the incoherent scattering in SANS experiments. $\mathrm{pH}$ was measured with a classical $\mathrm{pH}$-meter on the corresponding $\mathrm{H}_{2} \mathrm{O}$-based solutions. All samples, analyzed within 24 hours from their preparation, are prepared well above the SL cmc, which is reported to be between 0.02 and $0.25 \mathrm{mg} / \mathrm{mL} .{ }^{61}$ In particular, Imura et al. ${ }^{62}$ have reported a cmc value for the acidic form of SL of about $0.11 \mathrm{mg} / \mathrm{mL}$. As for the fully ionized form $(\alpha=1)$, we performed the measurement ourselves as cmc was never reported before under these conditions, to the best of our knowledge. Surface tension experiments (Figure S4 in the Supporting information and related discussion) in $\mathrm{H}_{2} \mathrm{O}$ as a function of the concentration of the fully-ionized sophorolipid form provide a cmc value of about $0.15 \pm 0.05 \mathrm{mg} / \mathrm{mL}$, which is slightly higher but still comparable within error with the corresponding protonated form.

Experimental techniques. Transmission Electron Microscopy under cryogenic conditions (Cryo-TEM) were run on two different microscopes, according to the final resolution. Lower resolution images in Figure 7 were taken on a FEI Tecnai 120 Twin microscope operating at $120 \mathrm{kV}$ (magnification 30.000 fold) and equipped with a high resolution Gatan Orius CCD 4k x 4k numeric camera. Higher resolution images in Figure 9 were obtained on a Jeol 2010F at the PFMU, Institut Pasteur (Paris, France). The microscope operates at $200 \mathrm{kV}$ and magnification was 80.000 fold. A Gatan ultrascan 4000 camera was used to acquire the image. On both microscopes, DigitalMicrograph ${ }^{\mathrm{TM}}$ software was used for image acquisition. Cryofixation was either done on a EMGP, Leica (Austria) instrument or on a home made cryo-fixation device. Liquid samples at desired concentrations were deposited on holey $(10 \mu \mathrm{m})$ carbon coated TEM copper grids (Quantifoil R2/2, Quantifoil, Germany). Excess of sample was eliminated and the grid was immediately blotted into liquid ethane. All grids were kept at liquid nitrogen during storage and throughout all experimentation. Sample was observed with a Gatan Cryoholder (Gatan 626DH, Gatan, USA). Surface tension $(\gamma)$ 
experiments were performed to evaluate the cmc using a Tracker tensiometer, from Teclis (France), in the needle pendant drop configuration. Values are collected as a function of time and averaged after equilibrium is reached.

${ }^{1} \mathrm{H}$ solution NMR spectra were acquired on a Bruker AV300 Ultrashielded $($ Avance spectrometer equipped with a $5 \mathrm{~mm}(1 \mathrm{H} / \mathrm{BBF}) \mathrm{BBO}$ probe with Z-axis gradient using a standard $30^{\circ}$ flip angle; intermolecular contacts were studied from a series of ${ }^{1} \mathrm{H}-{ }^{1} \mathrm{H}$ Nuclear Overhauser Effect SpectroscopY (NOESY) NMR experiments with mixing times from 50 to $900 \mathrm{~ms}$, performed with a standard three-pulse sequence. ${ }^{45}$

Small Angle Neutron Scattering (SANS) was performed at the Léon Brillouin Laboratory (Orphée Reactor, Gif-sur-Yvette, France) on the PAXE beamline. The spectrometer configuration was adjusted to cover two different $q$-ranges. The small angle region $8.38 \cdot 10^{-3}$ $\AA^{-1}<\mathrm{q}<7.32 \cdot 10^{-2} \AA^{-1}$ is obtained with a neutron wavelength, $\lambda$, of $6 \AA$ and a sample-todetector distance, $\mathrm{D}$, of $5 \mathrm{~m}$. An acquisition time of $4800 \mathrm{~s}$ was used; the medium angle region covers a q-range $3.50 \cdot 10^{-2} \AA^{-1}<\mathrm{q}<2.80 \cdot 10^{-1} \AA^{-1}$ at $\lambda=6 \AA$ with $\mathrm{D}=1.20 \mathrm{~m}$. An acquisition time of $1800 \mathrm{~s}$ was used in that case. Wavevectors lower than $8.38 \cdot 10^{-3} \AA^{-1}$ were obtained using $\mathrm{D}=5 \mathrm{~m}$ and $\lambda=12 \AA$. $q$ is defined as $4 \pi / \lambda \sin \theta / 2$, where $\theta$ is the scattering angle between the incident and the scattered neutron beams. All samples are introduced in a 2 mm quartz cell and studied at $\mathrm{T}=22^{\circ} \mathrm{C}$. The blank sample is composed of $99.9 \% \mathrm{D}_{2} \mathrm{O}$, whose signal is subtracted from the experimental data. Data treatment is done with the PAsiNET.MAT software package provided at the beamline and available free of charge. ${ }^{63}$ Absolute values of the scattering intensity are obtained from the direct determination of the number of neutrons in the incident beam and the detector cell solid angle. The 2D raw data were corrected for the ambient background and empty cell scattering and normalized to yield an absolute scale (cross section per unit volume) by the neutron flux on the samples. The data were then circularly averaged to yield the 1-D intensity distribution, I(q). The incoherent scattering was approximated from the high- $q$ intensity plateau and subtracted from the corresponding reduced data. Finally, data collected at the different configurations were used together without the need of introducing a correction factor, given the same acquisition wavelength under both configurations. Scatter software ${ }^{64}$ was used to fit the form factor of selected SANS data sets using classical equations for sphere, cylinder, disk and vesicle models. ${ }^{65}$ Ellipsoidal shape was derived from the cylindrical form factor. All fits were executed considering an homogeneous core system. The relationship between the scattering wavevector, q, and distance is $d=\frac{2 \pi}{q}$. Relative considerations on the surface-to-volume 
ratio are done on the basis of the classical Porod law at high-q: ${ }^{65} I \underset{q=0}{I}(q)=\frac{2 \pi}{q^{4}} \cdot \Delta \rho \cdot \frac{S}{V}$, where $\mathrm{I}(\mathrm{q})$ is the SANS measured intensity in absolute scale, $\Delta \rho$ is the difference in the scattering length density between the solvent and the irradiated object, $\mathrm{S}$ is the total exposed surface, $\mathrm{V}$ is the total irradiated volume of all objects and $\phi$ is the volume fraction.

\section{Acknowledgements}

The research leading to these results has received funding from the European Community’s Seventh Framework Programme (FP7/2007-2013) under Grant Agreement $n^{\circ}$ Biosurfing/289219. G. Carrot (PAXE line, Laboratoire Léon Brillouin, Gif-sur-Yvette, France) for assistance on the PAXE beamline. M. Rawiso (Institut Charles Sadron, Strasbourg, France), M. Impéror-Clerc (Laboratoire de Physique du Solide, Université de Paris XII, Orsay) and F. Michaux (École Nationale Supérieure d'Agronomie et des Industries Alimentaires de Nancy, Nancy, France) are kindly acknowledged for helpful discussions on SANS. M. In (Laboratoire Charles Coulomb, Université de Montpellier II, France) is thoroughly acknowledged for his precious hints to interpret SANS data. M.-N. Rager (Ecole Nationale Supérieure de Chimie Paris, Paris, France) and F. Ribot (Laboratoire de Chimie de la Matière Condensée de Paris, Université Pierre et Marie Curie, Paris, France) are kindly acknowledged for helpful discussion on NMR experiments. A. Salonen (Laboratoire de Physique des Solides, Université d'Orsay, France) is kindly acknowledged for providing access and support to surface tension experiments.

\section{Supporting Information Available}

Figure S1: Results of the fit for SANS data of the $\mathrm{SL} / \mathrm{D}_{2} \mathrm{O}$ solutions. Table S1: Numerical results from fits of SANS. Figure S2: Results of the fit for SANS data for the $5 \mathrm{mg} / \mathrm{mL}$ and $\alpha=1$ system. Figure S3: Direct representation of the experimental structure factor and related discussion. Figure S4: surface tension experiments to determine the cmc value of the fullyionized $(\alpha=1)$ sophorolipid form. This material is available free of charge via the Internet at http://pubs.acs.org

\section{References}

\footnotetext{
${ }^{1}$ Xu, C.; Fu, X.; Fryd, M.; Xu, S.; Wayland, B. B. ; Winey, K. I. ; Composto, R. J. Reversible StimuliResponsive Nanostructures Assembled from Amphiphilic Block Copolymers Nano Lett. 2006, 6, 2282-287
} 
${ }^{2}$ Jacquin, M.; Muller, P.; Cotter, H.; Théodoly, O. Self-assembly of charged amphiphilic diblock copolymers with insoluble blocks of decreasing hydrophobicity Langmuir 2010, 26, 18681-18693

${ }^{3}$ Kawasaki, H.; Souda, M.; Tanaka, S.; Nemoto, N.; Karlsson, G.; Almgren, M.; Maeda, H. Reversible vesicle formation by changing $\mathrm{pH}$ J. Phys. Chem. B 2002, 106, 1524-1527

${ }^{4}$ Lin, Y.; Han, X.; Huang, J.; Fu, H.; Yu, C. A Facile Route to Design pH-responsive Viscoelastic Wormlike Micelles: Smart Use of Hydrotropes J. Coll. Interf. Sci. 2009, 330, 449-455

${ }^{5}$ Chu, Z.; Feng, Y. pH-switchable Wormlike Micelles Chem. Commun. 2010, 46, 9028-9030

${ }^{6}$ Negrini, R.; Mezzenga, R. pH-Responsive Lyotropic Liquid Crystals for Controlled Drug Delivery Langmuir 2011, 27, 5296-5303

${ }^{7}$ Ray, S.; Das, A. K.; Banerjee, A. pH-Responsive, Bolaamphiphile-Based Smart Metallo-Hydrogels as Potential Dye-Adsorbing Agents, Water Purifier, and Vitamin B 12 Carrier Chem. Mater. 2007, 19, 1633-1639

${ }^{8}$ Meister, A.; Bastrop, M.; Koschoreck, S.; Garamus, V. M.; Sinemus, T.; Hempel, G.; Drescher, S.; Dobner, B.; Richtering, W.; Huber, K. et al. Structure-Properties Relationships in Stimuli-Responsive Bolaform Hydrogels Langmuir 2007, 23, 7715-7723

${ }^{9}$ Meister, A.; Blume, A. Self-Assembly of Bipolar Amphiphiles Curr. Op. Colloid Interf. Sci. 2007, 12, 138-147

${ }^{10}$ Kjellin, M.; Johansson, I. Surfactants from Renewable Resources, John Wiley \& Sons, Ltd: West Sussex, 2010

${ }^{11}$ von Rybinski, W.; Hill, K. Alkyl Polyglycosides-Properties and Applications of a new Class of Surfactants Angew. Chem. Int. Ed. 1998, 37, 1328-1345

12 Stubenrauch, C. Sugar Surfactants - Aggregation, Interfacial, and Adsorption Phenomena Curr. Op. Coll. Interf. Sci. 2001, 6, 160-170

${ }^{13}$ Hoffmann, B.; Platz, G. Phase and Aggregation Behaviour of Alkylglycosides Curr. Op. Coll. Interf. Sci. 2001, 6, 171-177

${ }^{14}$ Baccile, N.; Nassif, N.; Malfatti, L.; Van Bogaert, I. N. A.; Soetaert, W.; Pehau-Arnaudet, G.; Babonneau, F. Sophorolipids: a Yeast-Derived Glycolipid as Greener Structure Directing Agents for Self-Assembled Nanomaterials Green Chem. 2010, 12, 1564-1567

${ }^{15}$ Rau, U.; Hammen, S.; Heckmann, R.; Wray, V.; Lang, S. Sophorolipids: a Source for Novel Compounds Ind. Crops Prod. 2001, 13, 85-92

${ }^{16}$ Tulloch, A. P.; Hill, A.; Spencer, J. F. T. Structure and Reactions of Lactonic and Acidic Sophorosides of 17Hydroxyoctadecanoic Acid Canad. J. Chem. 1968, 46, 3337-3351

${ }^{17}$ Asmer, H.-J. ; Lang, S.; Wagner, F.; Wray, V. Microbial Production, Structure Elucidation and Bioconversion of Sophorose Lipids. J. Am. Oil. Chem. Soc. 1988, 65, 1460-1466

${ }^{18}$ Zhou, S.; Xu, C.; Wang, J.; Gao, W.; Akhverdiyeva, R.; Shah, V.; Gross, R. Supramolecular Assemblies of a Naturally Derived Sophorolipid Langmuir 2004, 20, 7926-7932

${ }^{19}$ Develter, D. W. G.; Fleurackers, S. J. J. Surfactants from Renewable Resources, John Wiley \& Sons, Ltd: West Sussex, 2010; pp. 213-238

${ }^{20}$ Maingault, M. Use of Sophorolipids and Cosmetic and Dermatological Compositions, WO/1995/034282A

${ }^{21}$ Van Bogaert, I. N. A.; Saerens, K.; De Muynck, C.; Develter, D.; Soetaert, W.; Vandamme, E. J. Microbial

Production and Application of Sophorolipids Appl. Microbiol. Biotechnol. 2007, 76, 23-34

${ }^{22}$ Shete, A. M.; Wadhawa, G.; Banat, I. M.; Chopade, B. A. Mapping of Patents on Bioemulsifier and Biosurfactant: a Review J. Sci. Ind. Res. 2006, 65, 91-11

${ }^{23}$ Fu, S. L.; Wallner, S. R.; Bowne, W. B.; Hagler, M. D.; Zenilman, M. E.; Gross, R.; Bluth, M. H. Sophorolipids and Their Derivatives are Lethal Against Human Pancreatic Cancer Cells J. Surg. Res. 2008, 148, 77-82

${ }^{24}$ Ishigami, Y.; Gama, Y.; Nagahora, H.; Yamaguchi, M.; Nakahara, H.; Kamata, T. The pH Sensitive Conversion of Molecular Aggregates of Rhamnolipid Biosurfactant Chem. Lett. 1987, 763-766

${ }^{25}$ Kitamoto, D.; Morita, T.; Fukuoka, T.; Konishi, M.; Imura, T. Self-Assembling Properties of Glycolipid Biosurfactants and Their Potential Applications Curr. Op. Coll. Interf. Sci. 2009, 14, 315-328

${ }^{26}$ Masuda, M.; Shimizu, T. Lipid Nanotubes and Microtubes: Experimental Evidence for Unsymmetrical Monolayer Membrane Formation from Unsymmetrical Bolaamphiphiles Langmuir 2004, 20, 5969-5977

${ }^{27}$ By degree of ionization we refer to the ratio between $\mathrm{COO}^{-}$and $\mathrm{COOH}$ groups, as defined in Eq.1 and discussed in the related paragraph.

${ }^{28}$ Zimmels, Y.; Lin, I. J. Stepwise Association Properties of Some Surfactant Aqueous Solutions Coll. Polym. Sci. 1974, 252, 594-612

${ }^{29}$ Maeda, H.; Eguchi, Y.; Suzuki, M. Hydrogen Ion Titration of Oleic Acid in Aqueous Media J. Phys. Chem. 1992, 96, 10487-10491

${ }^{30}$ Bendejacq, D.; Joanicot, M.; Ponsinet, V. Pearling Instabilities in Water-Dispersed Copolymer Cylinders With Charged Brushes Eur. Phys. J. E 2005, 17, 83-92 
${ }^{31}$ Reiss-Husson, F.; Luzzati, V. The Structure of the Micellar Solutions of Some Amphiphilic Compounds in Pure Water as Determined by Absolute Small-Angle X-Ray Scattering Techniques J. Phys. Chem. 1964, 68, 3504-3511

${ }^{32}$ Ekwall, P.; Mandel, L.; Solyom, P. The Aqueous Cetyltrimethylammonium Bromide Solutions J. Coll. Interf. Sci. 1971, 35, 4, 519-528

${ }^{33}$ Quirion, F.; Magid, L. J. Growth and Counterion Binding of Cetyltrimethylammonium Bromide Aggregates at 25 Degree: a Neutron and Light Scattering Study J. Phys. Chem. 1986, 90, 5435-5441

${ }^{34}$ Goyal, P. S.; Dasannacharya, B. A.; Kelkar, V. K.; Manohar, C.; Srinivasa Rao, K.; Valaulikar, B. S. Shapes and Sizes of Micelles in CTAB Solutions Physica B 1991, 174, 196-199

${ }^{35}$ Kuperkar, K.; Abezgauz, L.; Danino, D.; Verma, G.; Hassan, P. A.; Aswal, V. K.; Varade, D.; Bahadur, P. Viscoelastic Micellar Water/CTAB/NaNO 3 Solutions: Rheology, SANS and Cryo-TEM Analysis J. Coll. Interf. Sci. 2008, 323, 403-409

${ }^{36}$ Glatter, O.; Fritz, G.; Lindner, H.; Brunner-Popela, J.; Mittelbach, R.; Strey, R.; Egelhaaf, S. U. Nonionic Micelles Near the Critical Point: Micellar Growth and Attractive Interaction Langmuir 2000, 16, 8692-8701

${ }^{37}$ Shrestha, R. G.; Shrestha, L. K.; Sharma, S. C.; Aramaki, K. Phase Behavior and Microstructures of Nonionic Fluorocarbon Surfactant in Aqueous Systems J. Phys. Chem. B 2008, 112, 10520-10527

${ }^{38}$ Hickl, P.; Ballauff, M.; Jada, A. Small-Angle X-ray Contrast-Variation Study of Micelles Formed by Poly(styrene)-Poly(ethylene oxide) Block Copolymers in Aqueous Solution Macromolecules 1996, 29, 40064014

${ }^{39}$ Zhang, R.; Marone, P. A.; Thiyagarajan, P.; Tiede, D. M. Structure and Molecular Fluctuations of n-Alkyl- $\beta$ d-glucopyranoside Micelles Determined by X-ray and Neutron Scattering Langmuir 1999, 15, 7510-7519

40 Milkereit, G.; Garamus, V. M.; Veermans, K.; Willumeit, R.; Vill, V. Structures of Micelles formed by Synthetic Alkyl glycosides with Unsaturated alkyl Chains J. Coll. Interf. Sci. 2005, 284, 704-713

${ }^{41}$ Sodermann, O.; Johansson, I. Polyhydroxyl-Based Surfactants and Their Physico-Chemical Properties and Applications Curr. Op. Coll. Interf. Sci 2000, 4, 391-401

${ }^{42}$ Kwon, S.Y. Length Control in Rigid Cylindrical Nanoassembly by Tuning Molecular Interactions in Aqueous Solutions Langmuir 2008, 24, 10674-10-679

${ }^{43}$ Shimizu, T.; Masuda, M.; Minamikawa, H. Supramolecular Nanotube Architectures Based on Amphiphilic Molecules Chem. Rev. 2005, 105, 1401-1443

${ }^{44}$ Imae, T.; Funayama, K.; Krafft, M. P.; Giulieri, F.; Tada, T.; Matsumoto, T. Small-Angle Scattering and Electron Microscopy Investigation of Nanotubules Made from a Perfluoroalkylated Glucophospholipid $J$. Colloid Interf. Sci., 1999, 212, 330-337

${ }^{45}$ Ernst, R. R.; Bodenhausen, G.; Wokaun, A. Principles of Nuclear Magnetic Resonance in One and Two Dimensions, Oxford University Press: New York, 1987

46 Macura, S.; Ernst, R. R. Elucidation of Cross Relaxation in Liquids by Two-Dimensional N.M.R. Spectroscopy Mol. Phys. 1980, 41, 95

${ }_{47}$ Zhao, J.; Fung, M. Conformational Change of Hydrocarbon Chains Upon the Formation of Micelles J. Phys. Chem. 1993, 97, 5185-5187

48 Yuan, H.-Z.; Cheng, G.-Z.; Zhao, S.; Miao, X.-J.; Yu, J.-Y.; Shen, L.-F.; Du, Y.-R. Conformational Dependence of Triton X-100 on Environment Studied by 2D NOESY and 1H NMR Relaxation Langmuir 2000, 16, 3030-3035

${ }^{49}$ Aydogan, N.; Abbott, N. L. Interfacial Properties of Unsymmetrical Bolaform Amphiphiles with One Ionic and One Nonionic Head Group J. Colloid Interf. Sci. 2001, 242, 411-418

${ }^{50}$ Kan, P. L.; Papahadjopoulos-Sternberg, B.; Wong, D.; Waigh, R. D.; Watson, D. G.; Gray, A. I.; McCarthy, D.; McAllister, M.; Scha1tzlein, A. G.; Uchegbu, I. F. Highly Hydrophilic Fused Aggregates (Microsponges) from a C12 Spermine Bolaamphiphile J. Phys. Chem. B 2004, 108, 8129-8135

51 The theoretical OA chain length is calculated with $l=1.5+1.265 n$, where $l$ is the chain length in $\AA$ and $n$ is the number of $\mathrm{CH}_{2}$ groups. This value is in agreement with the $24.22 \AA$, as reported in Ref. 16 .

52 Tanford, C. The Hydrophobic Effect: Formation of Micelle and biological Membranes, John Wiley \& Sons, Ltd: New York, 1980

53 Abel, S.; Dupradeau, F.-Y.; Raman, E. P.; MacKerell Jr., A. D.; Marchi, M. Molecular Simulations of Dodecyl- $\beta$-maltoside Micelles in Water: Influence of the Headgroup Conformation and Force Field Parameters J. Phys. Chem. B 2011, 115, 487-499

${ }^{54}$ Kaibara, K.; Iwata, E.; Eguchi, Y.; Suzuki, M.; Maeda, H. Dispersion Behavior of Oleic Acid in Aqueous Media: From Micelles to Emulsions Colloid Polym Sci 1997, 275, 777-783

${ }^{55} \mathrm{OM}$ - (cis-9-octadecenyl)-4-O-( $\alpha$-glucopyranosyl)- $\beta$ - $D$-glucopyranoside) - has a chemical structure very close to SL. 
${ }^{56}$ de Koster, C. G.; Heerma, W.; Pepermans, H. A. M.; Groenewegen, A.; Peters, H.; Haverkamp, J. Tandem Mass Spectrometry and Nuclear Magnetic Resonance Spectroscopy Studies of Candida bombicola Sophorolipids and Product Formed on Hydrolysis by Cutinase Anal. Biochem. 1995, 230, 135-148

${ }^{57}$ Das, S.; Bhirud, R. G.; Nayyar, N.; Narayan, K. S.; Kumar, V. V. Chemical Shift Changes on Micellization of Linear Alkyl Benzenesulfonate and Oleate J. Phys. Chem. 1992, 96, 7454-7457

${ }^{58}$ Lang, S.; Brakemeier, A.; Heckmann, R.; Spockner, S.; Rau, U. Production of Native and Modified Sophorose Lipids Chim Oggi-Chem. Today 2000, 18, 76-79

59 Davila, A.-M.; Marchal, R.; Vandecasteele J.-P. Sophorose Lipid Production from Lipidic Precursors Predictive Evaluation of Industrial Substrates J. Ind. Microbiol. 1994, 13, 249-257

${ }^{60}$ Rau, U.; Heckmann, R.; Wray, V.; Lang, S. Enzymatic Conversion of a Sophorolipid into a Glucose Lipid Biotechnol. Lett. 1999, 21, 973-977

${ }^{61}$ Develter, D. W. G. ; Lauryssen, L. M. L. Properties and Industrial Applications of Sophorolipids Eur. J. Lipid Sci. Technol. 2010, 112, 628-638

${ }^{62}$ Imura, T.; Masuda, Y.; Minamikawa, H.; Fukuoka, T.; Konishi, M.; Morita, T.; Sakai, H.; Abe, M.; Kitamoto, D. Enzymatic Conversion of Diacetylated Sophoroselipid into Acetylated Glucoselipid: Surface-Active Properties of Novel Bolaform Biosurfactants J. Oleo. Sci. 2010, 59, 495-501

${ }^{63}$ http://didier.lairez.fr/dokuwiki/doku.php?id=pasinet

${ }^{64}$ Förster, S.; Burger, C. Scattering Functions of Polymeric Core-Shell Structures and Excluded Volume Chains Macromolecules 1998, 31, 879-891

${ }^{65}$ Glatter, O.; Kratky, O. Small Angle X-ray Scattering, Academic Press: London, 1982 